\title{
La virtualización de la educación superior en América Latina: entre tendencias y paradigmas
}

\author{
Virtualization of Higher Education in Latin America: Between Trends and \\ Paradigms \\ María Elena Chan Núñez \\ Universidad de Guadalajara. México. \\ machan@ cencar.udg.mx
}

\begin{abstract}
Resumen
El artículo presenta un acercamiento al campo de conocimiento sobre la virtualización de la Educación Superior en América Latina, a partir de la revisión de sus fundamentos y tendencias expuestos en publicaciones emitidas en los últimos diez años por organismos y autores clave para la región.

Se expone un modelo analítico que permite reconocer algunas relaciones y oposiciones entre concepciones y prácticas enunciadas en los documentos de referencia. A partir del análisis de los resultados de esos diferentes ejercicios de interés prospectivo, se reflexiona sobre la convergencia entre un paradigma de gestión de la innovación educativa para el fortalecimiento institucional, y un paradigma eco sistémico sobre las TIC que ofrece diferentes escenarios futuribles.
\end{abstract}

\section{Palabras clave}

Educación virtual, tendencias, prospectiva, innovación educativa, paradigmas.

\begin{abstract}
This article presents an approach to the field of virtualization of Higher Education in Latin America, from the revision of its basis and the trends showed in educational agencies publications and regional key authors in the last ten years. This paper introduces an analytical model that recognizes important relationships and oppositions between concepts and practices inside the consulted bibliography: from the analysis of outcomes to the use of different prospective exercises. This work also offers a reflection on the convergence between a paradigm of management in educational innovation for institution strengthening and an ICT ecosystemic paradigm that offers other possible scenarios.
\end{abstract}

\section{Key words}

Virtual education, trends, foresight, educational innovation, paradigms.

\section{Introducción}

Los estudios descriptivos y prospectivos que permiten reconocer tendencias y futuro de la educación virtual en América Latina, utilizan diferentes metodologías pero, en general, suelen partir de la recuperación de visiones de los expertos involucrados en el campo. ¿Cuál es la relación que se establece entre los paradigmas o modelos que fundamentan las opiniones expertas y las tendencias? ¿Cuáles son las tendencias más influyentes y como pueden interpretarse a la luz de paradigmas visibles en el campo de la virtualización educativa? 
En relación al uso de los términos de corrientes, modelos, enfoques, tendencias y paradigmas, Morales Gómez (2013) explica:
"Siguiendo un orden de frecuencia en el uso de estos términos se escucha más a menudo la expresión corrientes pedagógicas, seguida de modelos pedagógicos, enfoques curriculares, tendencias pedagógicas y paradigmas educativos. Todo parece depender de la profesión de quienes utilizan estos vocablos. Así por ejemplo, los psicólogos prefieren hablar de corrientes, los epistemólogos de enfoques y paradigmas, los sociólogos e historiadores de tendencias y los pedagogos de modelos".

Thomas S. Kuhn, definió el paradigma como un conjunto de valores, convicciones, teorías, modos de plantear problemas y resolverlos, compartidos por una comunidad científica. El sistema de ideas compartido permanece mientras emerge otro con la fuerza suficiente como para sustentar su validez (Kuhn, La estructura de las revoluciones científicas., 1971).

El mismo Morales Gómez (2013), en relación al concepto de tendencia señala que las tendencias expresan demandas sociales. Las tendencias no tienen tradición ni posicionamiento, se expresan como proyectos de mediano y largo plazo. Pertenecen a la investigación prospectiva y pueden no tener sustento teórico o validación práctica.

Las condiciones para la innovación educativa se han favorecido en los últimos años, por convergencia de ideas originadas desde hace al menos dos siglos, con soportes y medios que hoy, no sólo las han hecho visibles, sino que han permitido su desarrollo y difusión de manera exponencial.

La enseñanza orientada por los intereses de los estudiantes y lo que se concibió como educación personalizada se impulsó desde el siglo XVIII con Herbart (1776-1841), y ya en el siglo XIX con educadores como María Montessori (1870-1952), John Dewey (E.U. 1859-1952), Decroly (1871-1932), y en la misma línea e introduciendo el uso de medios de información y comunicación con Celestine Freinet (1896-1966) (de Zubiría Samper, 2008). En conjunto estos educadores han sido reconocidos como impulsores de la "escuela nueva", y han configurado una línea paradigmática con educadores de todo el mundo que han actualizado sus métodos, pero han sostenido los principios.

Es así que en la educación mediada por TIC se pueden observar modelos y enfoques que provienen de diferentes tiempos históricos. Se puede decir que, en el campo de la educación, como seguramente sucede en otros campos de conocimiento, se gestan hoy iniciativas con apariencia de innovación que en realidad no lo son tanto. Se comparten sistemas de creencias de los que no necesariamente se sabe el origen.

La velocidad con que circula la información por los medios digitales hoy, convierte rápidamente en tendencia ideas que no necesariamente llegarán a constituir sistemas de conocimiento en un sentido paradigmático.

Un ejemplo de ello es el "aula invertida", "flipped classroom" en inglés (Flipped Learning Network, 2014). Si se compara la definición de Flipped learning y la relativa al método de Celestine Freinet, pedagogo del siglo XIX, se puede observar que no hay diferencia en sus principios:

La virtualización de la educación superior en América Latina: entre tendencias y paradigmas. María Elena Chan. 
El aula invertida es una postura pedagógica en el que la instrucción va del espacio de aprendizaje grupal al espacio de aprendizaje individual. El espacio grupal es transformado en un ambiente dinámico, interactivo y creativo en el que el educador funge como guía para la aplicación de conceptos de una materia. (Flipped Learning Network, 2014)

Celestine Freinet, a finales del siglo XIX y principios del XX, instrumenta una pedagogía basada en actividades prácticas y organización cooperativa del trabajo para el aprendizaje. Tal como en el aula invertida, las actividades prácticas para realizar en grupo necesitaban coordinar acciones, compartir conocimientos, indagaciones y la reelaboración de ideas. La construcción de conocimientos se tornaba obra colectiva. El educando se consideraba un sujeto social impulsado por el deseo de expresarse y comunicarse. (Cembrano, 2010)

La virtualización de la educación se presenta como un fenómeno en el que se actualizan métodos educativos cuyas posibilidades se ven ampliadas con el uso de las TIC, pero también es una megatendencia económica y cultural que rebasa el ámbito de la educación escolar.

La virtualización de las prácticas sociales se está dando en otros ámbitos como la salud, la urbanización, el cuidado del ambiente, el manejo de la energía, el gobierno, la organización social y en todos los campos científicos y profesionales. (Mateos, García, \& González, 2015)

Mateos, García y González (2015) analizaron doce estudios de megatendencias realizados en diferentes partes del mundo y una mega-tendencia transversal a todos los ámbitos de la actividad humana es la virtualización, así como la consideración de inteligencia, entendida ésta como la capacidad de utilizar información y conocimiento para la toma de decisiones en cualquier campo de actividad humana.

"Sin duda, el incremento del grado de virtualización viene desde fines de los noventa y está relacionado con la digitalización global de la economía y la sociedad y con su impacto en el aprendizaje, debido a su mayor eficiencia en la capacidad de retención de aprendizajes al incorporar la diversidad de recursos de aprendizaje que favorecen los ambientes virtuales y, sobre todo, la flexibilidad y los menores costos de oportunidades de las personas" (Rama, 2012)

La virtualización vista como megatendencia trasciende la digitalización de las prácticas escolares para su operación a través de lo que se conoce como campus y aulas virtuales. Virtualizar supone hoy, la gestión del entramado entre los entornos de aprendizaje físicos y digitales, considerando que en unos y en otros hay mediación de las TIC para la representación o evocación de los objetos de conocimiento y el modelamiento de las interacciones para el aprendizaje.

"En el contexto tecnocultural actual, la relación entre el mundo real y el mundo virtual no puede entenderse como un vínculo entre dos mundos independientes y separados, que eventualmente coinciden en un punto, sino como una cinta de Moebius, donde no existe un adentro y un fuera, y donde es imposible identificar límites entre ambos. Para las nuevas generaciones, cada vez más, la vida digital se fusiona con su domesticidad como un elemento más de la naturaleza". Dolores Reig (SITEAL, 2014)

La virtualización de la educación superior en América Latina: entre tendencias y paradigmas. María Elena Chan. 
La virtualidad se vive no sólo en el universo que se abre tras la pantalla de la computadora, sino en todas las interfaces que se encuentran dispuestas en los entornos de vida de los seres humanos y en el uso de todo tipo de artefactos: aparatos portables, dispositivos en los automóviles, pantallas gigantes en las plazas comerciales, los tableros de aviso de los horarios en que pasará la ruta del transporte público, enseres domésticos, etcétera.

La virtualización educativa no está limitada a la representación de objetos, escenarios y experiencias del mundo real para colocarlos en entornos digitales, eso es sólo una dimensión de la virtualidad. También se opera en el modo inverso, en el que la mediación tecnológica está interviniendo los objetos y espacios físicos convirtiéndolos en dispositivos de información, conocimiento y comunicación. Visto así, no es que la vida tienda a transcurrir en los entornos digitales, es que los entornos natural y urbano (Echeverría, 2000) crecen en inteligencia computacional y posibilidades comunicativas articulando el entorno digital a los ecosistemas en los que se habita. (Pata, 2009)

En este artículo se presenta una lectura sobre formas de explicación del fenómeno de virtualización de la educación superior en América Latina considerando:

- Una reflexión sobre la construcción del campo de conocimiento de la educación mediada por TIC y los paradigmas visibles en su configuración.

- Reconocimiento de métodos y herramientas conceptuales para interpretar tendencias en la virtualización educativa.

- Análisis de algunos referentes sobre tendencias en la educación a distancia y aprendizaje en línea en América Latina.

\section{La construcción del campo de conocimiento de la educación mediada por TIC}

"Educación mediada por TIC" es probablemente la etiqueta temática más general que puede aplicarse a prácticas y líneas de investigación y desarrollo con diferente historia, enfoques, influencia y niveles de difusión tales como: la educación a distancia, el aprendizaje en línea, las modalidades educativas híbridas o mixtas, así como diferentes movimientos y propuestas didácticas recientes que se basan en el uso de TIC.

Este conjunto de prácticas educativas mediadas por TIC diferenciadas en su evolución, enfoques, métodos, y grados de institucionalización ¿pueden reconocerse como un solo campo de conocimiento? Si se toma la definición de campo de Pierre Bourdieu (Bourdieu, 1994), un campo se constituye cuando confluyen intereses de un grupo, prácticas similares, interacciones entre sujetos que se relacionan por su saber, juegos de poder que se originan por las diferentes posiciones no sólo relativas al saber, sino a lo que se instituye y reconoce a través de sujetos y organizaciones con influencia unas sobre otras.

Para el ejercicio reflexivo que se propone en este artículo la respuesta es afirmativa: si puede considerarse que la educación mediada por TIC y por ende el fenómeno de virtualización pertenecen a un campo de conocimiento en construcción permanente. Campo y paradigma no son sinónimos, un campo de conocimiento puede estar atravesado por múltiples paradigmas, pero lo que lo configura son objetos de investigación compartidos y la tensión entre esos paradigmas.

La virtualización de la educación superior en América Latina: entre tendencias y paradigmas. María Elena Chan. 
Las prácticas educativas mediadas por TIC han sido multidisciplinarias por naturaleza. Los distintos campos disciplinarios han aportado conceptos, teorías, métodos y paradigmas. Entonces, la educación mediada por TIC no es sólo un campo de conocimiento, sino también un campo que surge de la intersección de otros campos de conocimiento.

\section{Una propuesta analítica para visualizar el campo de conocimiento de la educación mediada por TIC}

El reconocimiento de paradigmas en sucesión, es un modo de lectura del devenir en un campo de conocimiento. El problema en el caso de la educación mediada por TIC, es que los paradigmas son múltiples, procedentes de las distintas disciplinas que atraviesan el campo, y no siempre convergentes en el mismo momento histórico.

Se está utilizando aquí el concepto de paradigma en dos sentidos: como eje ordenador de elementos que pertenecen a una misma clase, es decir, como categoría que emerge por el reconocimiento de algo que liga a estos elementos a manera de un patrón o modelo reconocible, y en su acepción de paradigma científico en tanto principios compartidos por una comunidad disciplinar, y en este caso multi o interdisciplinar, que suceden a un modelo explicativo anterior y significan por ello un modo alterno de explicación. (Kuhn, La estructura de las revoluciones científicas., 1971)

Como primer ejercicio para realizar una lectura que permitiera contrastar y en su caso diferenciar paradigmas del campo de la educación mediada por TIC, respecto de tendencias visibles en el campo de la virtualización educativa, se retomó el estudio de prospectiva realizado en el ILCE, Instituto Latinoamericana de Comunicación Educativa en 2008. (Miklos \& Arroyo, 2008) En un artículo elaborado por los autores de este estudio prospectivo, se exponen veintiséis variables que caracterizan y tendrán impacto en el futuro de la educación a distancia y el e-learning en América Latina.

A continuación se presenta un cuadro elaborado a partir de las áreas de conocimiento de las que se presume proceden las variables identificadas en el estudio de prospectiva al que se ha hecho referencia.

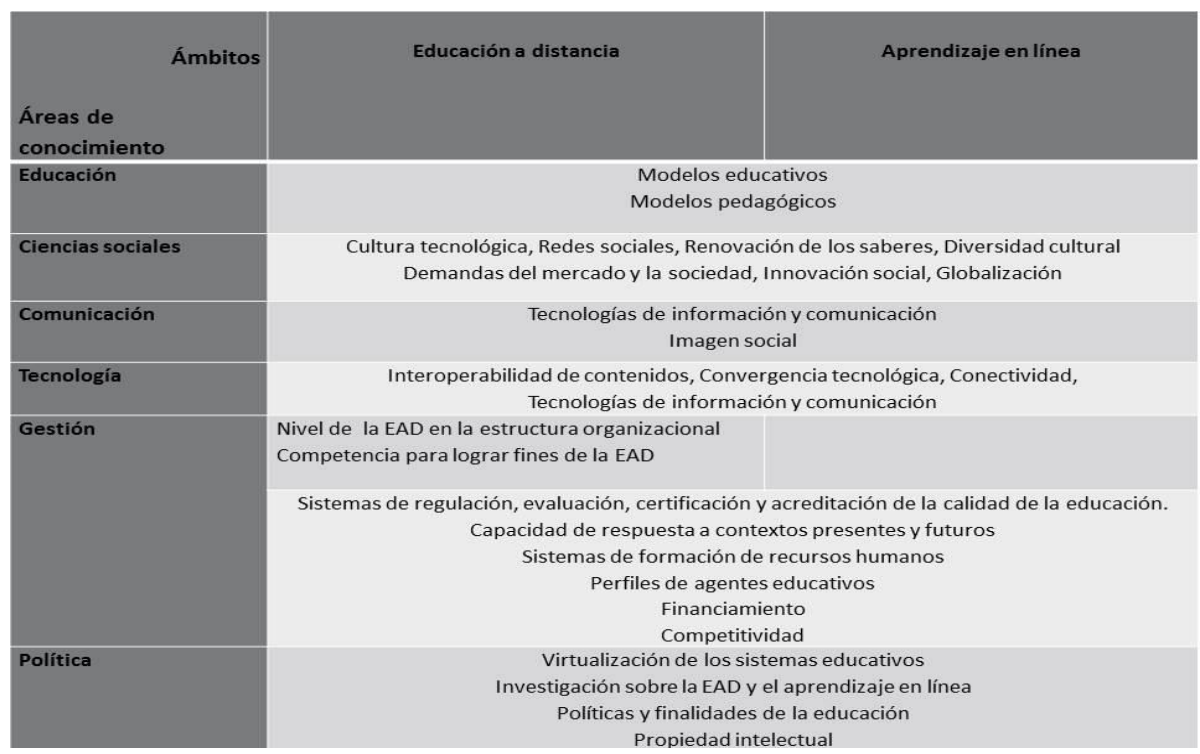

Cuadro 1- Variables identificadas en estudio prospectivo (Miklos \& Arroyo, 2008)

La virtualización de la educación superior en América Latina: entre tendencias y paradigmas. María Elena Chan. 
En el cuadro (1) se han integrado las veintiséis variables reconocidas en el estudio de Miklos y Arroyo (2008), y puede observarse que la mayor parte de estas se concentran en las áreas de gestión y política.

Las variables consideradas en esta prospectiva, relacionadas con educación y tecnología se mencionan de un modo genérico. Aparecen como categorías los términos de "modelos educativos y pedagógicos" y "tecnologías de información y comunicación".

Como resultado del estudio, Miklos y Arroyo (2008) formulan líneas estratégicas, y entre ellas se encuentran: "Diseño de un modelo educativo que sustente el aprendizaje virtual" y "Construcción, implantación y evaluación de modelos pedagógicos acordes con el aprendizaje individual, independiente y autogestivo". Respecto a las tecnologías apuntan otra línea estratégica: "Apropiación sociocultural de los elementos tecnológicos y digitales" y la otra alusión a la tecnología dentro de las líneas estratégicas es: "Desarrollo de la infraestructura tecnológica, conectividad y acceso con calidad y equidad” (Miklos \& Arroyo, 2008, pág. 37).

Lo primero que llama la atención en el planteamiento de las líneas estratégicas es que se haga referencia a "un modelo educativo", hablando en singular. Lo segundo es que pareciera reconocerse que el "aprendizaje virtual" requiere de un tipo de fundamentación específica, como si los fundamentos educativos previos a la emergencia de las TIC no fueran suficientes o adecuados. Y por otro lado se espera lograr, mediante algún tipo de formación, la "apropiación sociocultural de los elementos tecnológicos y digitales". No se integran ambas líneas estratégicas en una sola, porque al parecer, existe un modo de pensar las TIC como elementos independientes de los modelos educativos, aunque de alguna manera, también se aspira a generar un modelo acorde al contexto social mediado por las TIC.

La visión que separa lo educativo de lo tecnológico se ve reforzada en los resultados del estudio: "a pesar de la diversidad de enfoques, se podrían agrupar dos visiones sobre el porvenir de la educación a distancia y el e-learning y una mezcla de ambas". (Miklos \& Arroyo, 2008)

- Las visiones optimistas centradas en el desarrollo de las TIC a las que se les atribuye la energía creativa que provocará la transformación de la educación y la cultura. El aprendizaje se convertirá en abierto, flexible, colaborativo y contribuirá a que se cumplan los objetivos educativos universales.

- Las visiones escépticas que parten de reconocer una contradicción básica entre las fuerzas productivas de la sociedad y las innovaciones tecnológicas que les son propias y la organización social que marcha a la zaga de éstas. Para los escépticos el e-learning ahondará la desigualdad: "Esta tendencia no variará en el futuro y se crearán dos mundos educativos: uno que produce y distribuye cursos y otro que sólo consume, con las consecuencias obvias". (Miklos \& Arroyo, 2008) Así mismo desde estas visiones se incrementará la dependencia hacia modelos educativos que serán traspasados a través de las TIC.

En el modo de plantear las líneas estratégicas parece resolverse la tensión entre optimistas y escépticos: se recomienda fomentar la apropiación de los elementos tecnológicos y digitales, pero al mismo tiempo lograr modelos educativos y pedagógicos que impidan que

La virtualización de la educación superior en América Latina: entre tendencias y paradigmas. María Elena Chan. 
se traspasen los modelos ajenos, inherentes a la propia tecnología impuesta hegemónicamente.

Las visiones que se reconocieron en este estudio prospectivo realizado entre 2006-2007 en el ILCE, seguramente reflejan las perspectivas disciplinarias de los expertos involucrados, así como las posiciones desde las que han operado la educación a distancia y el e-learning.

Ejercicios como éste, en el que participan expertos y gestores, permiten ver escenarios posibles y reconocer la influencia de las dimensiones desde las que se piensa el futuro. En este caso el peso mayor se encuentra en las dimensiones de gestión y política y esto se manifiesta por la proporción de indicadores que se concentran en esas áreas.

¿Qué otras lecturas sobre paradigmas se pueden hacer si se toma como objeto el campo de la virtualización educativa a partir de su composición multidisciplinaria? ¿Qué otras visiones pueden ser recuperadas poniendo el acento en la multiplicidad de teorías, modelos, métodos y estrategias que se han originado en los distintos ámbitos de la educación mediada por TIC?

\section{Una lectura paradigmática de los modelos detrás de la virtualización de la educación}

García Aretio (2012) al expresar su visión sobre la educación a distancia y en el afán de hacer un aporte "integrador y global", explicita que no ha partido de cero y enfatiza como a mediados de los 80s, Keegan (1986, en García Aretio 2012) clasificó las teorías sobre la educación a distancia en tres grupos: las relativas a la autonomía e independencia; las teorías de la industrialización y las relativas a interacción y comunicación. A continuación se presenta un cuadro en el que se utilizan las categorías que retoma García Aretio de Keegan, sumando su propio agrupamiento realizado en Aretio (2001).

La matriz representa un cruce de aportaciones teóricas, las más sólidas a decir de García Aretio en el campo de la educación a distancia, y una interpretación de lo que estas categorías pueden significar hoy, considerando ámbitos de la educación mediada por TIC que apenas asomaban a principios de este siglo. Se retoma la actualización que el mismo García Aretio hace en su propuesta teórica a la que denomina "Diálogo didáctico mediado", en la que alude a la relación histórica entre medios y procesos educativos.

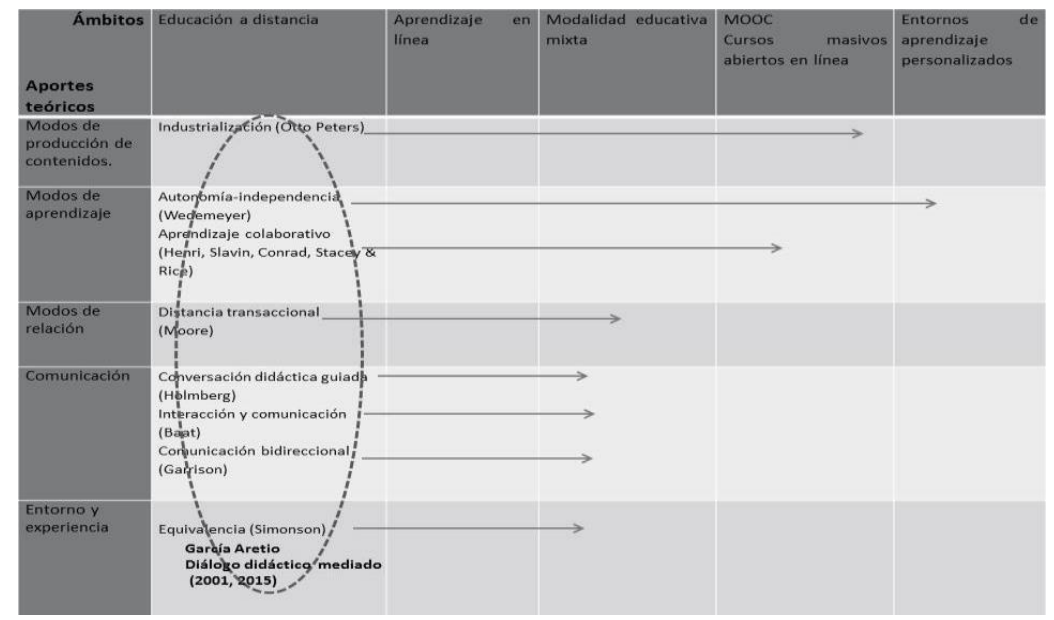

Cuadro 2 Diálogo didáctico mediado, integración teórica sobre EAD de García Aretio $(2001,2015)$ y sus alcances hacia otros ámbitos de la educación mediada por TIC.

La virtualización de la educación superior en América Latina: entre tendencias y paradigmas. María Elena Chan. 
En el cuadro (2) se han integrado categorías analíticas procedentes de la articulación teórica que hace García Aretio en torno a elementos centrales de la educación a distancia y con los cuales genera su propia propuesta a la que denomina "Diálogo didáctico mediado". La propuesta de García Aretio (2012) recupera la historicidad de las TIC y las coloca como parte de una explicación sobre posibilidades dialógicas diferenciadas por el uso de los diferentes dispositivos tecnológicos. En el cuadro se muestra lo que podrían ser alcances, representados por flechas, de la teoría del diálogo didáctico mediado, hacia otros ámbitos de la educación mediada por TIC.

Cabe notar que para el ámbito de los entornos de aprendizaje personalizados (PLE Personal Learning Environment), colocado en la cuarta columna del cuadro (2), los enfoques centrados en la relación docente-estudiante no aplican. En sentido estricto, la personalización de los entornos de aprendizaje (aunque haya enfoques que parten de la inducción docente o institucional para su desarrollo), es un proceso centrado en los intereses del estudiante, en su autonomía y en su capacidad de gestión de información y relaciones para aprender construyendo su propio ambiente de aprendizaje. (Adell \& Castañeda, 2013)

La extensión de teorías que han sido influyentes en una modalidad como la EAD, hacia otras modalidades o ámbitos, tiene que ver con el peso de sus conceptos esenciales y la posibilidad de reconocer su continuidad, en este caso, en diferentes tipos de prácticas educativas mediadas por TIC.

Así como es posible reconocer una relativa continuidad paradigmática, también es posible reconocer la aportación a los ámbitos de la educación mediada por TIC de otras disciplinas.

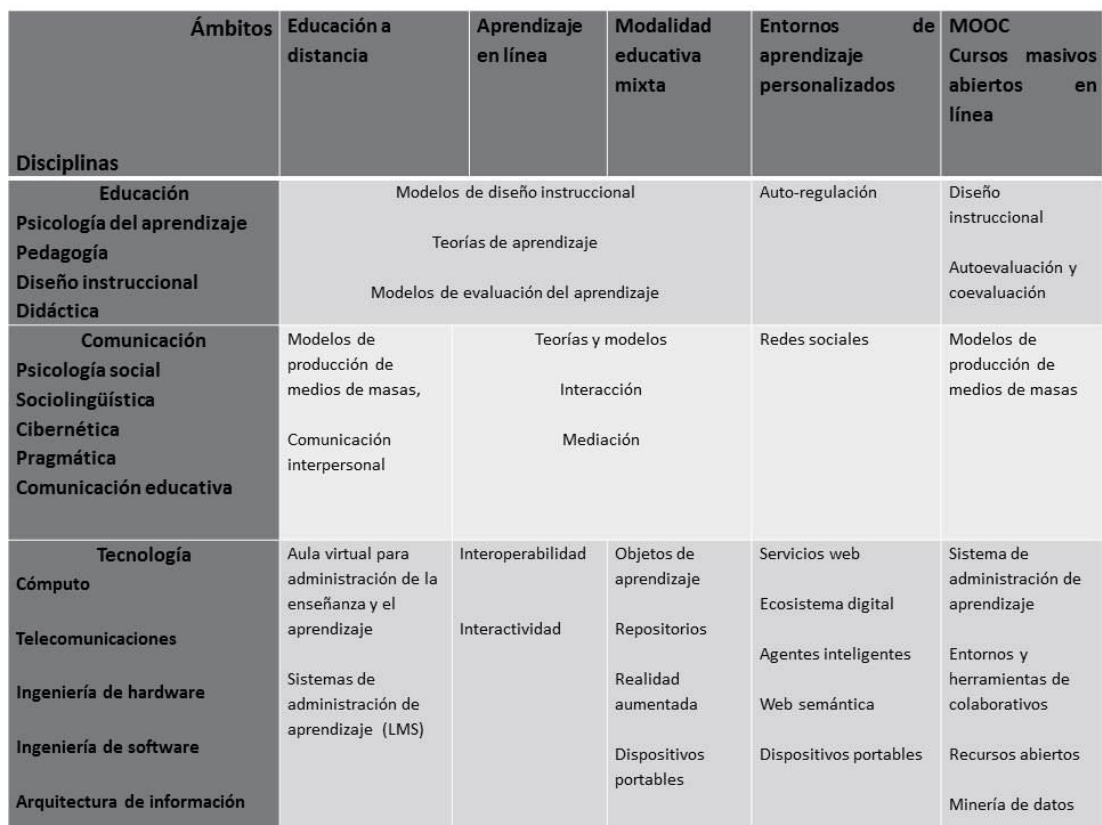

Cuadro (3) Matriz multidisciplinaria sobre el campo de la educación mediada por TIC

En el cuadro (3) se han dispuesto conceptos que tienen en este ejercicio una función categorial. En el eje horizontal se encuentran los mismos ámbitos de la educación mediada por TIC considerados en los cuadros (1) y (2), pero en el eje vertical se han

La virtualización de la educación superior en América Latina: entre tendencias y paradigmas. María Elena Chan. 
dispuesto campos de conocimiento que a su vez contienen distintas disciplinas, desde las que abrevan los desarrollos teóricos generados para el campo en su conjunto.

El cuadro es meramente enunciativo, pero muestra un ejercicio en el que lo que se quiere hacer notar es, que la configuración de la práctica educativa en cualquiera de los ámbitos, no depende exclusivamente de la fundamentación educativa o pedagógica, sino del entramado multi e interdisciplinario. Por dar un ejemplo: el concepto de Sistema de administración del aprendizaje LMS (Learning Managment System) requirió de la aplicación de métodos computacionales y de ingeniería de software, pero su composición ha dependido de una determinada manera de comprensión de lo escolar.

Si se cruza información entre el cuadro (1) y el (3), se puede reconocer que el aprendizaje colaborativo, retomado en 2001 por García Aretio como un concepto fundante en su propia teorización sobre la educación a distancia, deviene de autores que lo trabajaron cuando aún no emergía la Web colaborativa como tal. Pero sin duda los entornos digitales colaborativos han venido a profundizar el sentido del proceso de colaboración para el aprendizaje.

A su vez, fundamentos teóricos reconocidos por Keegan (1986) y retomados por García Aretio (2001, 2012), como la autonomía / independencia (desarrollados por autores como Wedemeyer y Saba en Aretio 2012) adquieren en el caso latinoamericano una connotación distinta. La educación a distancia en América Latina se vio fuertemente influida por principios provenientes de la educación popular de los años 70s, (principalmente del pensamiento de Paulo Freire) (Victoria, 2012); la cual pugnaba, desde asociaciones civiles hasta organismos internacionales como la UNESCO, por una cobertura universal, para lo cual la modalidad se consideró idónea.

Los planteamientos de Paulo Freire referidos a la autogestión en el aprendizaje conciben la autonomía e independencia no sólo respecto al formador, sino en un sentido más amplio, relativo a los sistemas políticos opresivos propios de las dictaduras de la época, sobre todo en América Central y del Sur.

Por las condiciones económicas latinoamericanas, el surgimiento de la modalidad educativa a distancia está ligado a la búsqueda de inclusión social, y esto llevó a la construcción de modelos basados en principios de educación popular tales como la horizontalidad, la formación basada en los intereses comunitarios, la colaboración, la investigación acción y la autogestión como principal aspiración en los procesos formativos.

Otro ejemplo de continuidad paradigmática puede verse en el otro extremo del cuadro (3) y en relación a los cursos masivos abiertos. En el recuadro puede observarse una conexión con el modelo industrial de producción de contenidos de la educación a distancia que fue reconocido en la teoría crítica de Otto Peters (1993). El concepto de MOOC al que se refiere la matriz es el más difundido y no el original concebido como cMOOC basado en el enfoque conectivista, del cual se hablará más adelante.

El modelo de instrucción basado en la producción industrial de contenidos con escaso o nulo margen de personalización de las actividades y sus productos, contrasta con propuestas de diseño para entornos constructivistas (Jonassen, 2000), que han generado otro tipo de estrategias basadas en el aprender haciendo y en la colaboración grupal.

La virtualización de la educación superior en América Latina: entre tendencias y paradigmas. María Elena Chan. 
Otra cuestión que interesa resaltar sobre el ejercicio analítico de la matriz multidisciplinaria, sobre la que se configura el campo de la educación mediada por TIC, es que deliberadamente se ha hecho una enunciación de disciplinas vinculadas a la Educación, la Comunicación y la Tecnología, porque suele nombrarse cada una de estas áreas de conocimiento, de manera monolítica, como si no se tuvieran en su interior constructos teóricos provenientes de disciplinas diversas.

El saber tecnológico es, probablemente, el que menos se reconoce en su configuración multi e interdisciplinaria. Suele pensarse en la tecnología como un solo tipo de saber, y muy poco se reflexiona sobre paradigmas en el propio desarrollo tecnológico que son convergentes o no con los paradigmas educativos o comunicativos.

Un ejemplo de choque paradigmático elemental puede observarse en el uso que se da a los teléfonos celulares en un aula convencional. La toma de fotografía de una pizarra llena de notas del profesor suele provocar reacciones críticas con frases tales como "ya ni se toman la molestia de escribir". Pero detrás de este uso de la herramienta fotográfica, lo que está es una práctica vertical en la que el apunte docente constituye el contenido sobre el que versará un examen o sobre el que habrá que realizar determinada tarea. El problema no reside en que la tecnología facilite la toma de nota de algo interesante para el estudiante que quiere guardar para hacer algo con eso, sino el hecho de que represente la misma práctica de toma de apuntes que se convertirán en contenido único de evaluación de una determinada asignatura escolar.

La innovación en el campo de la educación mediada por TIC ha evolucionado de un modo no lineal y probablemente los choques paradigmáticos entre disciplinas y enfoques son lo que caracteriza el dinamismo en su construcción. A su vez puede observarse que la EAD ha fungido como paradigma sobre los otros diferentes ámbitos de la educación mediada por TIC:

"De forma tradicional la EAD primero, el elearning después, y en general la ayuda tecnológica al aprendizaje que hasta ahora ha habido en distintas modalidades, han tenido un denominador común, han supuesto romper barreras de distinta naturaleza, no solo de lugar también las de tiempo, y las originadas por toda clase de problemas que impiden o dificultan la concurrencia de alumnos y profesores en un lugar físico en un intervalo de tiempo. También supuso la superación de déficits personales y de discapacidades". (Zapata-Ros, 2013)

La impronta que la EAD ha puesto en la educación en general tiene que ver, sobre todo, con la ruptura de barreras espacio temporales para la escolarización. El tipo de esfuerzos para la inclusión social se ha trasladado también a la inclusión de personas con déficits biopsicosociales: escolarizar sin límites de tiempo o distancia ha sido la consigna.

Marlene M. Blois (2012) ofrece otra mirada en la que el paradigma se da en sentido contrario: "la desescolarización" como movimiento, haciendo un recuento de los esfuerzos de la educación popular por el reconocimiento de "la escuela sin puertas ni ventanas" en el Brasil de finales de los años 70s. El sueño se hizo realidad con la mediación tecnológica, porque se logró llevar oferta educativa más allá de los edificios escolares. La autora narra el tránsito del uso de los medios masivos para llegar educativamente a la población abierta. La educación se llevó a las corporaciones, como un modo de reconocer la posibilidad de realizar la educación fuera del contexto escolar.

La virtualización de la educación superior en América Latina: entre tendencias y paradigmas. María Elena Chan. 
Un problema para visualizar y decir algo sobre el futuro de la educación, y más específicamente, de la educación virtual, es que ante la diversidad de paradigmas, e incluso la convergencia de paradigmas que resultan contradictorios o disonantes según el campo disciplinario del que provienen, es difícil afirmar que existe un sistema de creencias, prácticas y saberes integrados.

En los cuadros (1) (2) y (3) se incluyeron sólo algunas categorías aplicadas en la teorización de la educación a distancia, y trasladadas hacia otros ámbitos de la educación mediada por TIC. La educación a distancia, a su vez, se puede observar como paradigmática para el aprendizaje en línea y las modalidades educativas mixtas, e incluso en alguna medida para los MOOC, porque entre todos esos ámbitos se comparten principios que emanaron de sus teorías fundamentales, y han sido usadas con mayor o menor grado de consenso en diferentes organizaciones.

Otro autor contemporáneo de gran influencia en el campo del diseño educativo, Charles Reigeluth (2012), alude a un paradigma aún más amplio, opuesto al paradigma industrial de producción de la educación a distancia observado críticamente por Otto Petters, y es el de la educación post-industrial, y para la cual señala, se requiere una teoría instruccional alternativa. Reigeluth (2012) explica que el paradigma instruccional que se desarrolló en la era industrial se caracterizó por la selectividad diferenciadora entre los estudiantes que debieran hacer el trabajo manual respecto a aquellos que podrían llegar a una formación profesional. Hoy esa selectividad ya no es necesaria si se considera que el conocimiento es la base de la economía, y lo que se requiere es la formación de sus profesionales. El paradigma rebasa la escala de los modelos pedagógicos, porque se remite a una escala institucional en la que reconoce al sistema educativo como diseñado para la selección y no para el aprendizaje. De ahí que modelos postindustriales deban orientarse a maximizar el aprendizaje.

Reigeluth (2000) compiló diferentes modelos de instrucción como parte una revisión de teorías y prácticas de referencia. En la introducción de la obra expresó su concepto de circunstancias educativas: la naturaleza de lo que se va a aprender, la naturaleza del alumno, la naturaleza del ambiente de aprendizaje, y de sus limitaciones como el tiempo, los recursos, etcétera.

Entre los modelos integrados por Reigeluth en su obra Teorías y modelos de la instrucción, destacan cuatro que aluden al concepto de entorno de aprendizaje: Hanaffin (2000) quien expone el concepto de entornos de aprendizaje abiertos, Jonassen (2000) dedicado a los entornos constructivistas de aprendizaje, Schank, Berman y Macpherson (2000), con los simuladores de aprendizaje práctico y Pogrow (2000) con el uso sistemático de entornos de alta estimulación. Estos cuatro autores integran teorías y métodos procedentes del cómputo, la psicología cognitiva, y la didáctica. Consideran como espacio de aprendizaje tanto la interfaz del ordenador como el aula. Así mismo le dan un alto valor a la interacción entre los educandos y al uso del conocimiento ante una actividad demandante de su aplicación.

Este tipo de planteamientos coinciden en cuanto a los principios de aprendizaje contextualizado y colaborativo, con el paradigma de la comunicación educativa impulsado en América Latina desde los años 70s, aplicado tanto a la educación a distancia como a la educación popular, que ha tenido entre su exponentes a autores como Daniel Prieto, Francisco Gutiérrez, Jorge Huergo, y Mario Kaplún. (En Chan 2006)

La virtualización de la educación superior en América Latina: entre tendencias y paradigmas. María Elena Chan. 
Las teorías y modelos de la instrucción recuperados por Reigeluth (2000) y los mencionados como parte del paradigma de la comunicación educativa en Chan (2006), no tienen referencias cruzadas, pero pueden situarse en un sistema de conocimiento más amplio que los incluye: la postura constructivista frente al conocimiento, considerando que ésta se caracteriza por postular que el conocimiento se construye a través de la actividad de los aprendientes, que son éstos los que generan el significado, que el aprendizaje requiere la resolución de problemas con estrategias propias de pensamiento, y que la formación debiera organizarse a partir del conocimiento previo poseído por los educandos. (Arnold Cathalifaud, 2004)

El constructivismo es un paradigma que se ha fortalecido por la emergencia de las TIC, y a su vez, y de modo circular, uno de los más influyentes en la construcción de la noción de entorno virtual. Su naturaleza es transversal, ha signado prácticas educativas presenciales, formales, no formales y ahora virtuales. En sus orígenes se encuentra la epistemología genética de Piaget, centrado en la relación del individuo y su medio, así como la postura constructivista social de Vigotsky.

Desde la perspectiva de Arnold Cahtalifaud, en el constructivismo hay posturas "blandas" y "duras". Las posturas blandas son las que se han difundido y apropiado más extensamente en los sistemas educativos convencionales y han influido también a la educación mediada por TIC. Las posturas duras han sido asumidas por autores como Maturana (1997), quien a su vez ha influido en autores que han promovido un conjunto de principios explicativos sobre el conocimiento en las redes digitales y que se conocen como "Conectivismo" (Downes, 2012).

El estatus teórico del Conectivismo se encuentra en un provocativo punto de debate, y ha sido contra-argumentado por autores como Zapata-Ros (2015). Más allá del estatus teórico de la propuesta, destacan entre los principios conectivistas cuatro conceptos con un fuerte potencial heurístico para explicar el aprendizaje en el contexto social mediado por TIC: apertura, diversidad, interacción y autonomía. Estos cuatro conceptos son transdisciplinarios, y se encuentran asociados a la epistemología sistémica y ambiental.

Una categoría más inclusiva aún, que puede dar sentido a un sistema de conocimiento que agrupa posturas constructivistas y conectivistas, es el ecosistema. El paradigma ecosistémico en la educación mediada por TIC, agrupa teorías de diferentes disciplinas, y puede aplicarse a todos los ámbitos de virtualización educativa, desde la educación a distancia hasta los entornos personales de aprendizaje. Este paradigma emergente alude a fundamentos epistémicos, teóricos y metodológicos aplicables a las circunstancias propias de la virtualidad. En este paradigma los problemas y objetos de conocimiento se construyen sobre los entornos, las interacciones, los procesos adaptativos, la configuración de comunidades, entre otros conceptos ambientalistas.

En la siguiente figura se representa la línea paradigmática que atraviesa diferentes tipos de teorías.

La virtualización de la educación superior en América Latina: entre tendencias y paradigmas. María Elena Chan. 


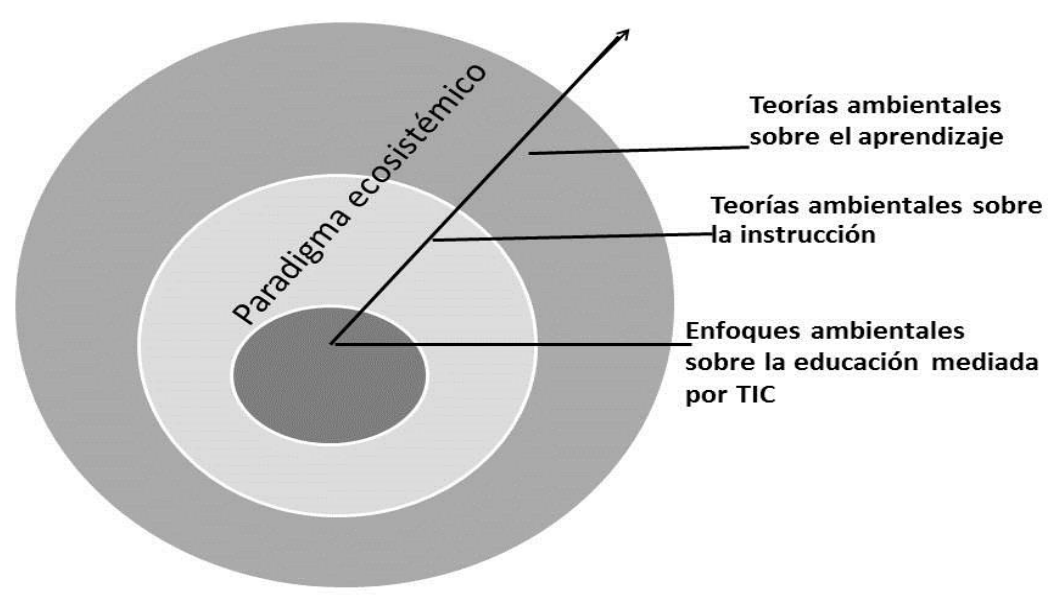

Figura 1. Paradigma ecosistémico desde las teorías de aprendizaje

Las teorías que parten de la noción de interacción con el entorno como base del aprendizaje, en el contexto de la educación virtual, problematizan tanto las interacciones que se generan en los entornos digitales, como las interacciones mediadas por las TIC en los entornos de vida físicos, considerando los dispositivos tecnológicos como parte del ecosistema (Pata, 2009)

Las teorías ambientalistas se sustentan en teóricos constructivistas como Piaget (1978), Vigotsky (1978), Maturana (1997), y autores constructivistas contemporáneos como (Jonassen, 2000), (Hannafin , Land, \& Oliver, 2000), (Pogrow, 2000), (Giraldo, 2012), y (Pata, 2009), entre otros.

La visualización de paradigmas que surgen a la par del fenómeno de virtualización educativa, puede parecer una tarea interpretativa que no tiene fin, porque las disciplinas no se detienen en la producción de nuevos conocimientos, y las convergencias paradigmáticas inter y transdisciplinarias no son fáciles de sostener, ni son explícitas en los ejercicios prospectivos en los que participan expertos en gestión y política educativa.

En esta primera parte del artículo se ha mostrado la complejidad de las relaciones paradigmáticas del campo de conocimiento de la educación mediada por TIC, en contraste con la escasa densidad que alcanza el manejo de los conceptos de Modelos educativos, Modelos pedagógicos y Tecnologías de información y comunicación, en tanto variables enunciadas al mismo nivel que las variables de gestión política y administrativa de modalidades educativas, que muestran, de acuerdo a las conclusiones de un primer estudio prospectivo, una función determinante en la construcción de los escenarios futuros, tomando como referencia, el estudio prospectivo de Miklos y Arroyo (2008).

El tejido interdisciplinario entre el campo de la educación mediada por TIC y los campos de conocimiento de la gestión administrativa y política pueden llevar a diferentes tipos de estrategias.

La virtualización de la educación superior en América Latina: entre tendencias y paradigmas. María Elena Chan. 


\section{Algunos referentes sobre tendencias en la virtualización educativa en América Latina}

En el 2012 se pidió a veinte autores de diferentes partes del mundo que expusieran su visión sobre la EAD desde una perspectiva histórica que enlazara pasado, presente y futuro para integrar un libro conmemorativo de los veinte años de iniciada la Educación a Distancia en la Universidad de Guadalajara en México (Moreno Castañeda, 2012). No se pretende sintetizar la riqueza de la diversidad y profundidad de esas visiones, pero analizando los discursos de los autores, se han distinguido algunos ejes que pueden resultar útiles para el análisis de tendencias en la virtualización de la educación superior en la región, considerando a la Educación a Distancia como modalidad influyente en los procesos de innovación educativa.

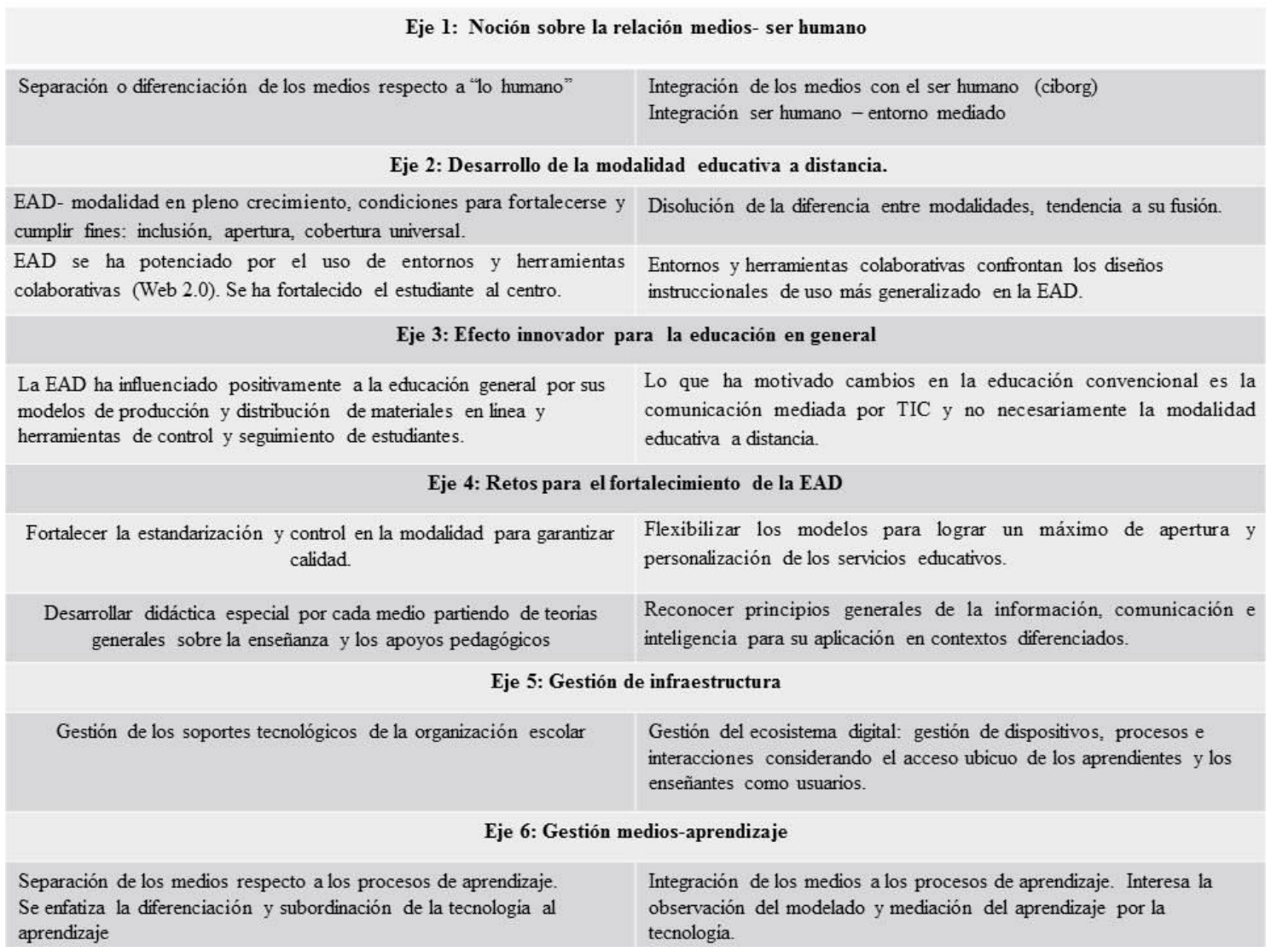

Cuadro 4- ejes de oposición identificados en visiones sobre la EAD

El cuadro sintetiza rubros en los que se concentraron las exposiciones de los autores del libro de referencia (Moreno Castañeda, 2012), considerando sólo aquellos en los que se encontraron posiciones encontradas. No todos los autores se expresaron en relación a los rubros identificados como polarizados, y hubo ejes con mayor número de aportaciones. Lo dicho por cada autor representa un universo discursivo que amerita ser leído como unidad. Sin embargo, para un ejercicio panorámico como el que se presenta en este artículo, parece útil esquematizar puntos en oposición para encontrar algún tipo de caracterización.

Puede observarse en el esquema, que aplicando la noción de paradigma como ordenador de elementos de la misma naturaleza, el polo que se integra en la columna izquierda, se orienta al fortalecimiento institucional de la modalidad educativa a distancia en los

La virtualización de la educación superior en América Latina: entre tendencias y paradigmas. María Elena Chan. 
planos: teórico, metodológico, estratégico, organizacional y tecnológico. Los planteamientos contenidos en ese eje en sentido vertical, son favorables a la institucionalización creciente de la modalidad, no sólo para su propio fortalecimiento, sino incluso para su transferencia hacia otras prácticas educativas que requieren el modelo de innovación que se considera propio de la EAD. Para efectos de este ejercicio analítico a este eje se le denomina "paradigma institucional".

La alineación de la columna derecha, presenta una visión en la que las modalidades diferenciadas pierden sentido, se apuesta por el desarrollo interdisciplinario de constructos teóricos, métodos y procedimientos de la educación virtual. Se opta por un manejo integral en el que la tecnología es parte del saber requerido para la gestión educativa. Aparecen conceptos que aluden al espacio, las interacciones, entornos y ecosistemas. Como parte de este ejercicio analítico, a esta alineación se le denomina "paradigma ecosistémico".

Estos dos ejes paradigmáticos servirán como guía para el análisis de estudios descriptivos y prospectivos que permiten reconocer tendencias en la virtualización de la educación en América Latina.

Las políticas educativas en América Latina en relación a TIC se han diferenciado en cada país, pero tienen en común su dinamismo (SITEAL, 2014). Ese dinamismo en la región en torno a la gestión de políticas públicas, se evidencia por la realización de estudios cooperativos en torno a la educación mediada por TIC y las consecuentes recomendaciones para su desarrollo.

En este apartado se presentan estudios realizados por diferentes grupos de trabajo que han tomado la Educación a distancia o al Aprendizaje en línea como objetos de descripción, análisis y prospección.

Se tomó como punto de partida el estudio prospectivo antes citado de Miklos y Arroyo, realizado por el Instituto Latinoamericano de Comunicación Educativa entre 2006 y 2007 (publicado en 2008); y se identificaron otros referentes comparables:

- El informe sobre Concepto y tendencias de la Educación a Distancia en América Latina auspiciado por el Centro de Altos Estudios Universitarios de la Organización de Estados Iberoamericanos y la Agencia Española de Cooperación Internacional para el Desarrollo AECID, publicado en 2009.

- La investigación colectiva sobre Educación Superior a Distancia en América Latina y el Caribe: realidades y tendencias coordinado por Claudio Rama y Patricia Lupión, auspiciado por la organización Virtual Educa, la UNISUL de Brasil y la Pontificia Universidad Católica de Puerto Rico, en el que autores de doce países hicieron análisis de sus sistemas educativos a distancia;

- El informe sobre Tendencias sociales y educativas 2014 con énfasis en Políticas TIC del SITEAL, Sistema de Información sobre Tendencias Educativas en América Latina.

- Y el libro sobre las Nuevas fronteras de la educación a distancia publicado en 2015 por el Instituto Latinoamericano y del Caribe de Calidad en Educación Superior a Distancia conjuntamente con el Observatorio para la Educación a Distancia de la organización Virtual Educa.

La virtualización de la educación superior en América Latina: entre tendencias y paradigmas. María Elena Chan. 
Se procedió a hacer una lectura comparativa de estos cinco referentes, considerando como categorías descriptivas, el ámbito de la educación mediada por TIC que tomaron por objeto; el método utilizado y las categorías o variables consideradas para sus respectivos procesos de análisis.

Se generaron cuadros de concentración para presentar la información de los distintos referentes de manera sintética utilizando ocho categorías:

- Tipo de resultado esperado en la propuesta

- Descripción o situación del ámbito educativo o modalidad de referencia

- Visión problemática

- Valores

- Retos

- Papel de la educación en el contexto social amplio

- Papel conferido a las TIC

- Consideraciones paradigmáticas

A continuación se comentan los cuadros de concentración de los estudios consultados. El eje horizontal de todos los cuadros de concentración se ordenó considerando la cronología de los referentes.

En el primer cuadro de concentración se presentan los principales descriptores de cada estudio, los que permiten identificar sus ámbitos de análisis, los métodos utilizados y lo que utilizan como categorías analíticas para la integración de la información, o las que resultan del procesamiento informativo.

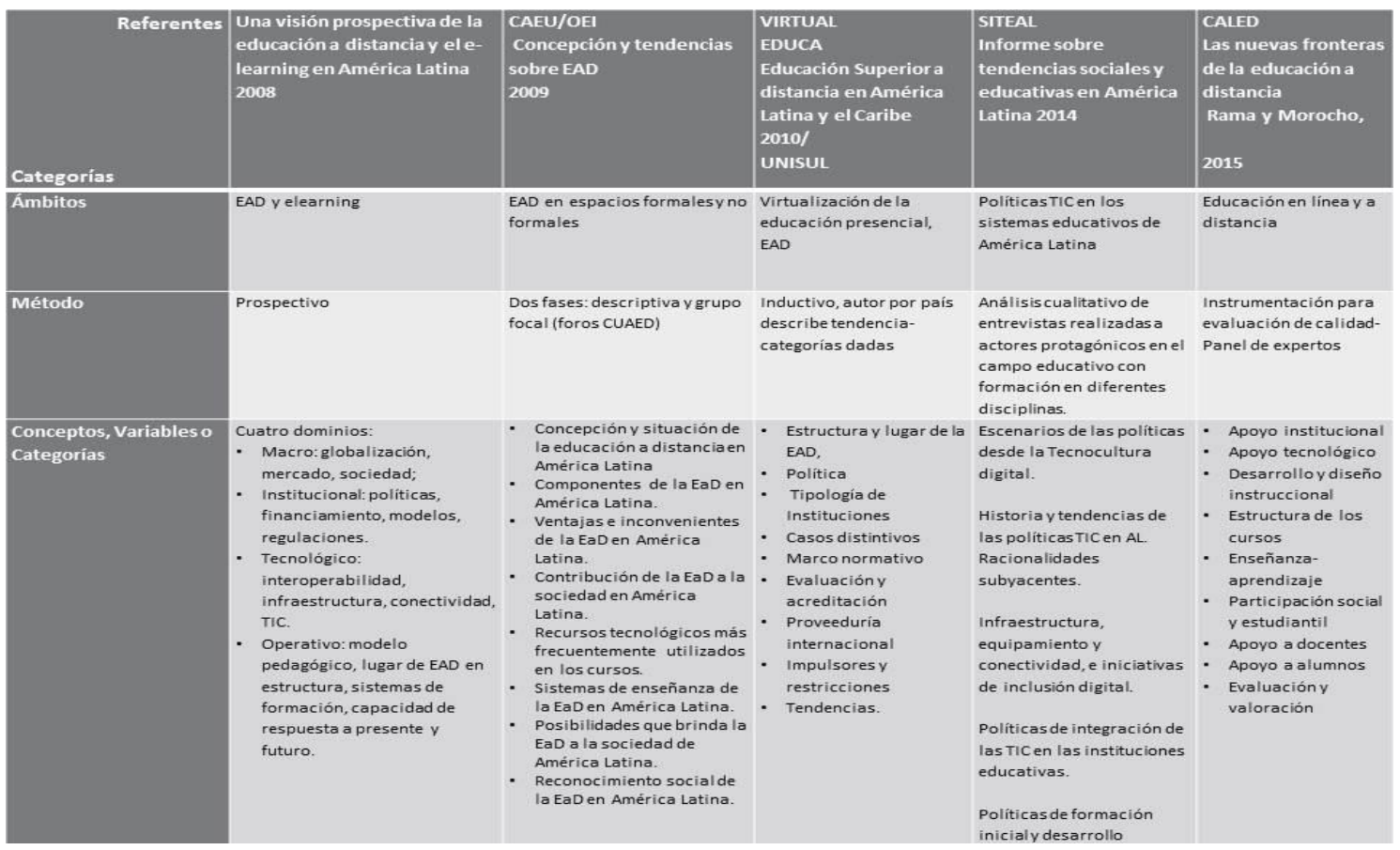

Cuadro 5- Descriptores básicos sobre referentes de tendencias en la educación mediada por TIC en América Latina

Cuatro de los estudios se orientan a la Educación a distancia. El informe de SITEAL es el único que tiene como referencia las políticas sobre TIC en un marco más general de tendencias sociales y educativas. Tanto el estudio prospectivo del 2008, como el desarrollado por CALED en 2015, para generar indicadores de calidad, además de la

La virtualización de la educación superior en América Latina: entre tendencias y paradigmas. María Elena Chan. 
educación a distancia toman como objeto el aprendizaje y la educación en línea, respectivamente.

Sobre los métodos utilizados, en todos los casos se hizo consulta a expertos, con diferentes tipos de procesamiento de información. El análisis prospectivo se aplicó en el caso del estudio realizado por el ILCE en 2008. A partir de la consulta a expertos se obtuvieron variables, que se integran en dominios, se extrajeron correlaciones y se hicieron consultas sucesivas hasta obtener escenarios.

Las unidades de análisis en el caso del CAEU/OEI (2009), se establecieron a partir de la participación en foros internacionales y por consulta a expertos que respondieron a preguntas genéricas sobre la modalidad a distancia. En este caso es significativo que una comunidad de práctica que interactúa cotidianamente en los foros impulsados por la Cátedra UNESCO de Educación a Distancia, se convierta en un universo de indagación sobre tendencias por el tratamiento temático que opera.

En todos los casos se observan conceptos que fueron utilizados categorialmente para prospectar o describir situación y tendencias del ámbito educativo de referencia.

En el caso del panel organizado por el CALED (2015) los indicadores para evaluar la calidad de la educación a distancia fueron resultado y no punto de partida, dado que de lo que se trató es justamente de lograr la instrumentación de un referente para la evaluación de la calidad de las modalidades educativas a distancia y en línea. Puede observarse que las variables obtenidas son aplicables a escala de programa institucional.

En el estudio prospectivo auspiciado por el ILCE (Miklos \& Arroyo, 2008), la tecnología aparece como uno de los dominios, al mismo nivel que las variables de carácter macro, las institucionales y las operativas. El modelo educativo forma parte del dominio operativo. Este mismo estudio prospectivo otorga un lugar preponderante, a las variables externas de carácter contextual. También el informe del SITEAL (2014) presenta un análisis contextual, y parte de la descripción de la "tecnocultura" como marco de las políticas en materia de TIC.

En el siguiente cuadro se presentan elementos que describen de manera sintética algunos de los hallazgos más importantes de cada tipo de estudio.

La virtualización de la educación superior en América Latina: entre tendencias y paradigmas. María Elena Chan. 


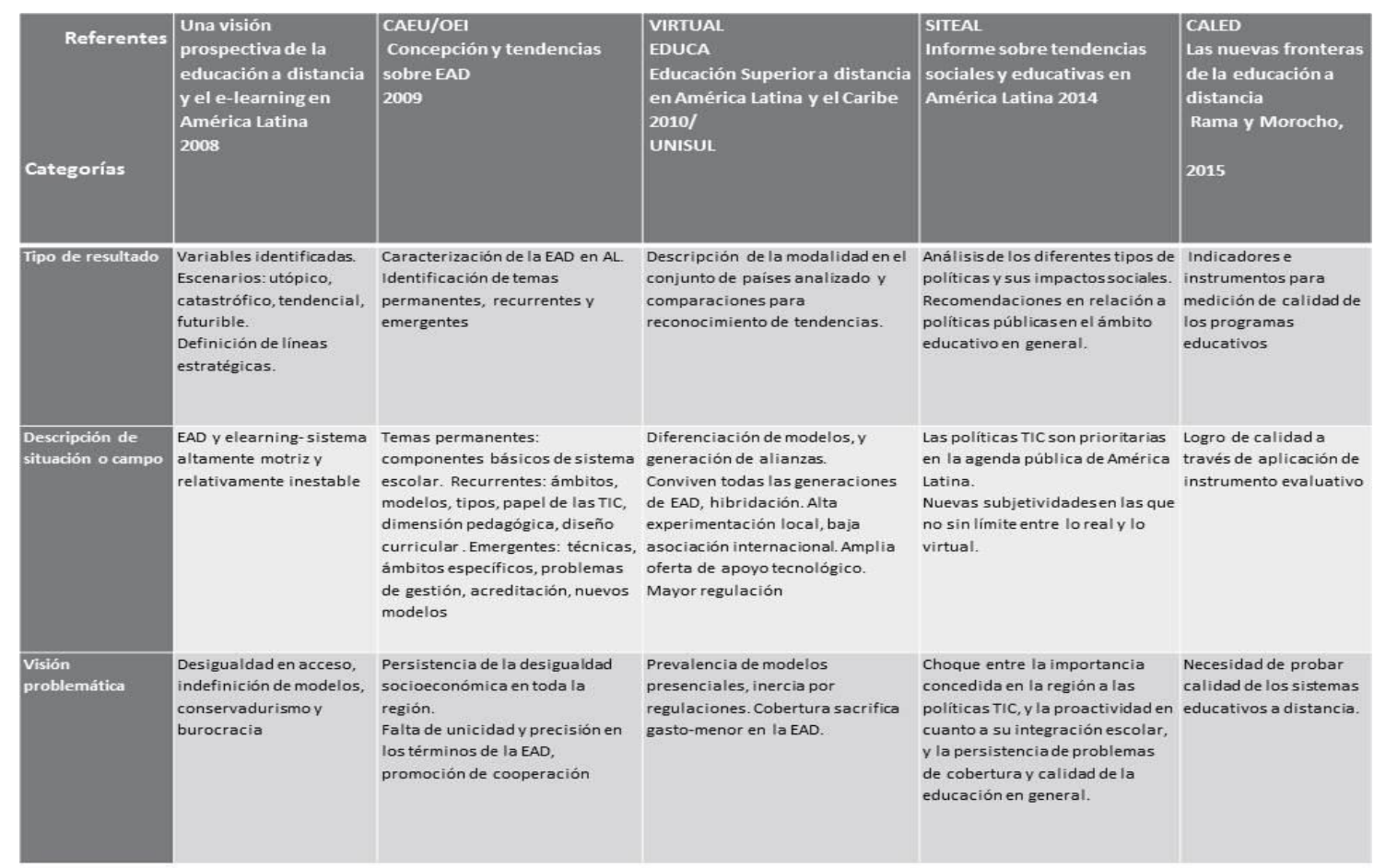

Cuadro 6- Referentes sobre futuro de la educación mediada por TIC en América Latina y descriptores cualitativos.

Por el tipo de resultado, en todos los casos, se logra no sólo algo que decir sobre la situación y las tendencias de la educación a distancia, el e-learning, la virtualización de la educación en general, o las políticas TIC, sino también herramientas conceptuales para guiar la observación y la acción a nivel institucional e interinstitucional.

En la parte baja del esquema, se puede observar cierta complementariedad entre los estudios en relación a la visión problemática. En dos de los referentes se encuentra la desigualdad económica como un problema estructural de la región. La cobertura como desafío en la región es también un elemento compartido.

En tres de los estudios: Visión prospectiva (2008), CAEU/OEI (2009) y el del SITEAL (2014), todos ellos orientados por una escala de observación regional, se alude a conservadurismo, inercias normativas así como choque entre proactividad en las políticas TIC contra problemas endémicos como la falta de cobertura y de calidad en la educación como sistema.

Afirman Miklos y Arroyo (2008) que los escenarios clave en la prospectiva son el escenario tendencial y el escenario futurible, dado que el primero es el más realista y el segundo el deseable-factible. Lo encontrado como tendencial en cuanto a la globalización creciente de la oferta educativa, la desigualdad en el acceso y la lentitud de las instituciones escolares para cambiar sus modelos, es coincidente con lo que aparece en las visiones problemáticas en los demás referentes considerados, como puede observarse en la tabla de concentración.

Un elemento mencionado en el estudio prospectivo de Miklos y Arroyo (2008) en relación a la constante revisión de los modelos educativos, como una acción siempre inacabada que no responde a tiempo a los cambios sociales, tiene su correlativo en las

La virtualización de la educación superior en América Latina: entre tendencias y paradigmas. María Elena Chan. 
visiones sobre la problemática expresados tanto en el informe del CAEU/OEI/AECID (2009), en donde se expresa como "falta de unicidad e imprecisión en los términos sobre EAD”.

Uno de los métodos utilizados por el estudio CAEU/OEI/AECID (2009) fue el análisis de los foros de la Cátedra Unesco de Educación a Distancia, lo que llevó al reconocimiento de los temas tratados en ese espacio. En los temas identificados, tanto en los recurrentes como en los emergentes, los modelos educativos se encuentran en constante discusión. Lo que se observa es que en los temas permanentes se encuentran categorías asociadas a componentes invariantes de la EAD, en los recurrentes se discuten los modelos, aquellos que son ya conocidos por la comunidad de práctica, y que los "nuevos modelos" se colocan en los temas emergentes.

Como espacio de observación de la dinámica entre los tipos de temas, los foros de comunidades de práctica, constituyen un espacio muy útil para identificar el cruce entre paradigmas (aquello que permanece y organiza estructuralmente la discusión), y las tendencias (lo que emerge y se sostiene de manera variable en tiempo, volumen de participación e incitación de expertos con menor o mayor influencia en la comunidad).

En el último cuadro de concentración se presentan categorías que representan el significado que se da a las modalidades educativas, a las TIC y lo que aparece como valores y retos enunciados como tales en los referentes. Lo que se declara como finalidad de la educación, de las modalidades y de los propios estudios realizados se asocia con valores. Las conclusiones y propuestas de los diferentes estudios o informes suelen formularse como retos o desafíos. Se integró la categoría de referencia paradigmática en la parte baja del cuadro, para comparar lo que en cada caso aparece como paradigma influyente o factor de innovación.

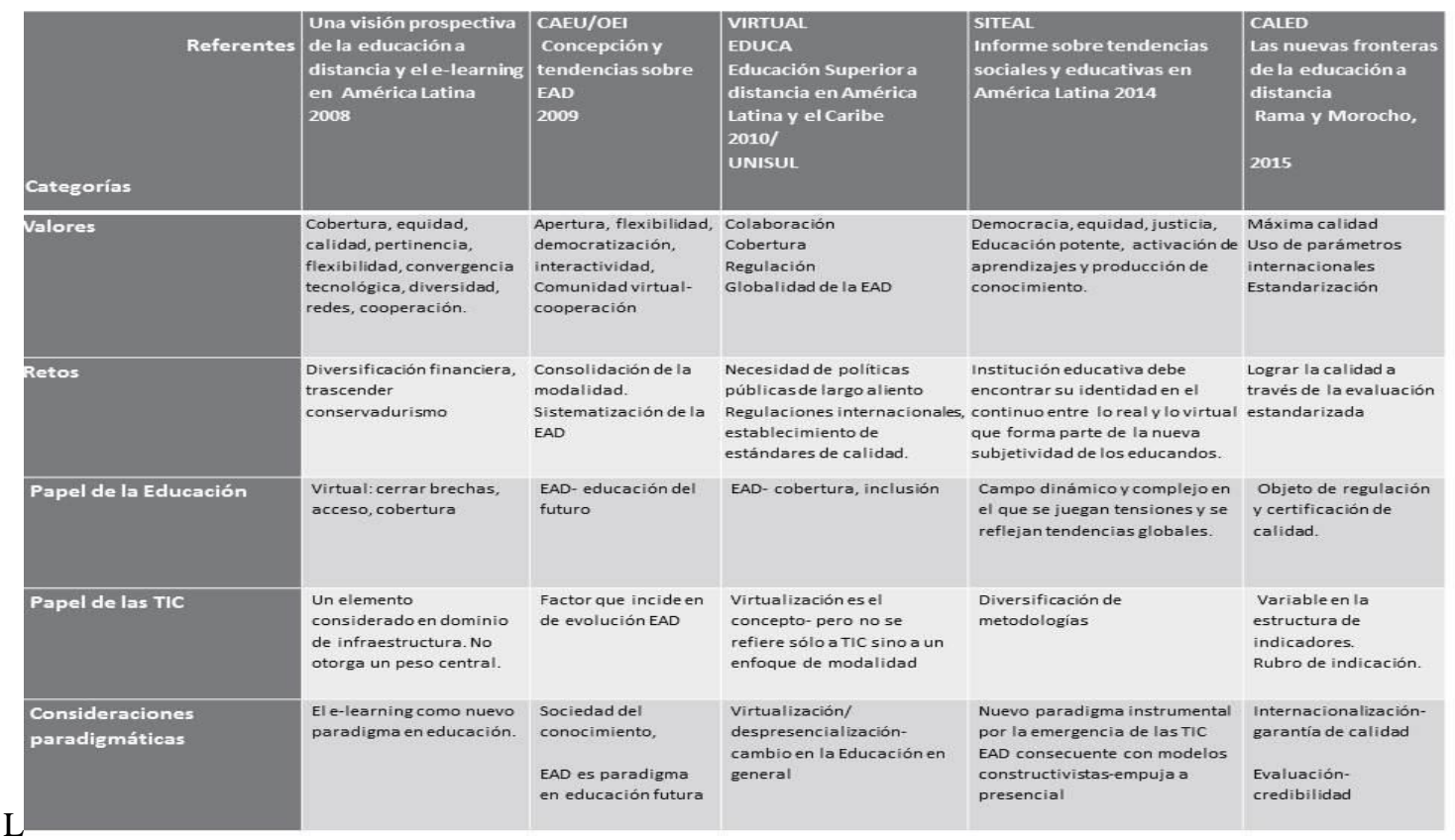

Cuadro 7- Referentes sobre futuro de la educación mediada por TIC en América Latina

Los valores que se comparten entre todos los estudios de referencia tienen que ver con equidad, cobertura, democracia y justicia.

La virtualización de la educación superior en América Latina: entre tendencias y paradigmas. María Elena Chan. 
Cooperación internacional es otro valor que repite en todas las instancias, así como la búsqueda de flexibilidad en los sistemas educativos y la calidad regulada por estándares compartidos.

Puede inferirse que en el nivel axiológico, hay alta coincidencia en las visiones, en la medida que el tipo de problemáticas relativas a la desigualdad social, inequidad en el acceso a la educación, y de acceso a la tecnología, han significado una motivación para la creación e impulso de la modalidad educativa a distancia y para las políticas de conectividad universal impulsadas en algunos países de la región.

En la línea de retos hay mayor variación en los enfoques, sobre los cuales es posible reconocer los paradigmas identificados antes: el instituyente y el ecosistémico.

Entre las líneas estratégicas definidas como resultado de la prospectiva de (Miklos \& Arroyo, 2008), se plantean acciones para fortalecer las modalidades educativas a distancia y en línea, desde la construcción de un modelo educativo propio de la educación virtual, formación y acompañamiento de los agentes, desarrollo de sistemas de información y evaluación, así como la apropiación sociocultural de los "elementos tecnológicos", entre otras. Las líneas estratégicas se orientan a la consolidación de las modalidades como medio para alcanzar un impacto positivo en la sociedad. El planteamiento en su conjunto se alinea con la centralidad de la institucionalidad de los procesos educativos y de su mediación tecnológica escolar.

En contraste el informe de SITEAL (2014) afirma que las instituciones educativas deben moverse hacia la nueva subjetividad de los educandos, lo cual tiene que ver con el modo como se observa la "tecno cultura" como contexto de las políticas y tendencias educativas. Esta consideración es asociada a una perspectiva ecosistémica porque deja de ver a las instituciones educativas y sus modalidades como instancias de cambio, y las coloca como parte de sistemas culturales más amplios en los que las TIC no son sólo herramientas para aprender, sino sobre todo, configuradoras de nuevas prácticas socioculturales.

El pensamiento de Dolores Reig, es recogido en el informe del SITEAL (2014) como nota de opinión sobre la información recabada a través de entrevistas con jóvenes en relación a significado de las TIC en sus vidas, e ilustra lo que se plantea aquí como una visión asociada al paradigma ecosistémico sobre la gestión de las TIC en educación:

"En el contexto tecnocultural actual, la relación entre el mundo real y el mundo virtual no puede entenderse como un vínculo entre dos mundos independientes y separados, que eventualmente coinciden en un punto, sino como una cinta de Moebius, donde no existe un adentro y un fuera, y donde es imposible identificar límites entre ambos. Para las nuevas generaciones, cada vez más, la vida digital se fusiona con su domesticidad como un elemento más de la naturaleza. En esta naturalización de la vida digital, los aprendizajes provenientes de ese entorno se mencionan con asiduidad, no solo porque se los pregunta explícitamente sino porque la referencia a Internet sale del modo más espontáneo entre los entrevistados."

Lo anterior contrasta con lo que se encuentra en la línea referida a retos y en la del papel de la educación, según puede verse en el cuadro (7): se apuesta a que a través de la educación, y más específicamente, a través de la modalidad educativa a distancia, se

La virtualización de la educación superior en América Latina: entre tendencias y paradigmas. María Elena Chan. 
logre cobertura, se cierren brechas socio-económicas, se logre inclusión social. Lo que aparece en el recuadro del SITEAL (2014) en estos aspectos es una formulación contrastante: el campo de la educación no se contempla como motor o instancia impulsora de cambios, es en sí misma un campo de tensiones en el que se reflejan las tendencias globales.

En la parte baja del cuadro (7), en el rubro de consideraciones paradigmáticas, puede observarse que cuatro de los referentes, la educación a distancia y la educación en línea se conciben como paradigma para el cambio de la educación en general. La excepción es el caso del informe del SITEAL (2014) donde el empuje para la innovación educativa se concibe ligado a la emergencia de las TIC. Puede asociarse el primer enfoque al paradigma institucional, porque se ve la EAD influyente, por trayectoria y consolidación. En cambio en el informe SITEAL las TIC aparecen como parte de un sistema cultural, con influencia contextual.

En resumen, considerando una lectura de contraste entre los referentes, puede reconocerse que la escala de observación regional, nacional o institucional, permite la identificación de tendencias utilizando diferentes métodos y definiendo también diferentes categorías analíticas, todas ellas de alto valor descriptivo y comprensivo para entender lo que está pasando en la educación a distancia y en la educación en línea.

La escala de observación regional, nacional o institucional no parece incidir en los enfoques que se asumen para generar las categorías y observables, y por ende, en los hallazgos y propuestas resultantes de los estudios, ya que se encuentran los mismos rubros independientemente de que las descripciones de la modalidad se apliquen a un país, o a programas institucionales como referentes particulares. Lo que parece diferenciar las posturas visibles en los informes, es el modo como se genera el tejido teórico metodológico, las dimensiones consideradas, las disciplinas desde las que se generan las observaciones y la fundamentación epistémica.

La trama presentada por el informe del SITEAL (2014) ilustra un modo de integración epistémica entre tendencias y paradigmas, porque coloca como objeto las Políticas TIC, pero su descripción y análisis toma como centro a los actores, y va hacia los jóvenes para recuperar sus usos y prácticas tecnológicas y las hace foco del análisis experto el cual abreva de la antropología, la comunicación, la psicología, la sociología, la ciencia política, por nombrar algunas de las disciplinas intervinientes. Las tendencias son observables en los descriptores de las políticas de manejo de las TIC, y su concreción, se hace visible en acciones como la gestión de la infraestructura y la emisión de políticas de formación docente, y para cada uno de estos componentes se hace la reflexión teórica desde constructos explícitos.

En todos los referentes comparados para este artículo, la metodología que utilizaron supuso que expertos de diversas disciplinas hicieran análisis de variables, contenidos, o llenaran descriptores, la diferencia, probablemente, estriba en el modo de presentación de los informes, pues sólo en el del SITEAL (2014), se explicita la fundamentación teórica tras la interpretación de los datos, y hubo una línea de construcción discursiva entre los expertos que intervinieron como investigadores cualitativos, analistas y comentaristas.

Las visiones epistémicas y teóricas están por supuesto detrás de las expresiones y elecciones de los sujetos convocados a ejercicios prospectivos (Miklos \& Arroyo,

La virtualización de la educación superior en América Latina: entre tendencias y paradigmas. María Elena Chan. 
2008), pero estos espacios no están organizados para el esclarecimiento de posturas, no es su finalidad.

\section{Articulación de tendencias y paradigmas}

En Margarita Victoria (2012) se observa una posición frente a la educación mediada por TIC en la que se intersectan la dimensión política, la pedagógica y la virtual: "la pedagogía de la virtualidad en su dimensión político-pedagógica es necesaria para generar una nueva gobernabilidad educativa en el ciberespacio".

Esta idea trasciende la visión de las TIC como herramienta de la educación a distancia o en línea y coloca las TIC como entorno en construcción para el que se requiere un tipo de formación particular. La autora lleva al plano de la experiencia en el ciberespacio la acción política. Trabaja con el concepto de rizoma para explicar una propuesta de pedagogía de la virtualidad. La política en Victoria (2012) no es una cuestión de gestión por autoridad, sino de participación de comunidades aprendientes.

Para dibujar un mapa sobre las tendencias en la virtualización de la educación, se requiere trabajar en el reconocimiento de los paradigmas teóricos multi e interdisciplinarios sobre los que se construye el campo de conocimiento. El problema, como se ha dicho antes, no está en la diversidad de propuestas y lucha de paradigmas, que le son propios a este campo de conocimiento, sino en el vacío de información sobre los impactos que los conceptos, enfoques y paradigmas tienen, al integrarse a un lenguaje de gestión y operación política.

Los MOOC son un ejemplo que ilustra como un concepto migra de un plano epistémico al político-económico. Los MOOC surgen como parte de una propuesta de experimentación conectivista. (Downes, 2012) Una de las interpretaciones que se da a la propuesta tiene que ver con el movimiento a favor del conocimiento abierto, y sus promotores en grandes universidades de prestigio se pronuncian a favor de la apertura de cátedras para cualquier persona interesada en ellas. Un paradigma epistémico se articuló con una visión empresarial cuando algunos de los autores participantes en el diseño de los primeros MOOC decidieron formar empresas para impartir ese tipo de cursos ante la enorme respuesta de usuarios de todo el mundo interesados en cursos prestigiados. (Bates 2014)

El concepto MOOC se ubica en diferentes paradigmas: el movimiento a favor del conocimiento abierto, el aprendizaje autónomo y personalizado, y también un enfoque de negocios basado en la gestión de conocimiento. La confusión y riesgo puede surgir si se eleva a política pública la pretensión de atender aspirantes a ingresar a la universidad con cursos masivos. También puede convertirse en un problema de gestión, el pretender que los docentes universitarios atiendan sus cursos regulares y además produzcan MOOC, sin que medie una política de apertura del conocimiento, o un plan de negocios claramente establecido, o la intención de desarrollar competencias colaborativas y de trabajo en red.

Considerar la adopción de estrategias formativas masivas, siguiendo modelos de producción industrial, o consumiendo la oferta de las grandes empresa transnacionales, cumple con uno de los descriptores del escenario catastrófico señalado en el estudio prospectivo de (Miklos \& Arroyo, 2008) en el que se advierte el riesgo de poner en

La virtualización de la educación superior en América Latina: entre tendencias y paradigmas. María Elena Chan. 
manos del mercado global la función educativa en la región, reduciendo el papel de los universitarios al consumo de la oferta formativa extranjera.

Otro ejemplo para reflexionar se presenta en el ámbito de los entornos personalizados de aprendizaje (PLE). La investigación básica y empírica en torno a la personalización de los entornos de aprendizaje, puede arrojar datos sobre la magnitud del acceso a los dispositivos, los usos educativos que se les da por parte de jóvenes estudiantes, y por otro lado, en el ámbito del desarrollo de aplicaciones para aparatos móviles, se puede observar una gama amplia de estrategias didácticas para todo tipo de objetos de conocimiento. (Adell \& Castañeda, 2013) La decisión sobre aplicación del gasto para el fortalecimiento de la infraestructura tecnológica con fines educativos en una determinada región, podría apostar por la dotación de equipos de cómputo para fortalecer el aprendizaje en escuelas, cuando probablemente, todo indique que sería más conveniente invertir en incrementar la conectividad pública gratuita y dotar de dispositivos móviles a la población infantil y juvenil en zonas económicamente deprimidas. Y esto no sólo tiene que ver con un compromiso para la reducción de la brecha digital garantizando acceso, sino sobre todo con una posición frente al aprendizaje en entornos abiertos, ubicuo y orientado a la personalización de los entornos para aprender.

En otra línea de desarrollo de la formación centrada en los intereses de los educandos, se encuentra el caso de los Appiarios, iniciativa de la organización Editacuja cuyos fundadores Martín Restrepo y Érica Casado, lo han aplicado en diferentes ciudades de Brasil y Colombia. (Editacuja, 2015) Los Appiarios, son espacios de emprendimiento social donde jóvenes, no necesariamente escolarizados, se forman en programación, diseño, gestión de contenidos y de negocios digitales. La metodología se basa en tres pilares: Descubrir (problemáticas), Experimentar (aprendiendo a generar ideas disruptivas) desarrollando proyectos, e Inventar, generando soluciones basadas en herramientas de prototipaje.

Este tipo de propuestas que se salen de la pauta de los espacios escolares convencionales, está irrumpiendo también en algunos sistemas educativos. La Universidad de la Sabana en Colombia se ha acercado para conocer el método de los Appiarios con la intención de generar uno en una comunidad aledaña al campus, extender con ello servicios de formación y brindar un espacio para la formación de universitarios bajo la estrategia abierta.

Otro caso es el de la Universidad de Guadalajara en México, en la cual se está llevando a cabo un proyecto en colaboración con la Universidad de Stanford centrado en la creación de Laboratorios de fabricación FabLab. La idea de los FabLab es propagar un movimiento formativo basado en proyectos de fabricación utilizando tecnologías de bajo costo. El laboratorio constituye un entorno creativo con tecnologías para la generación de objetos utilizando impresoras 3D, cortadoras láser y otras herramientas para materializar ideas creativas de los educandos. (Stanford University, 2015)

Aunque estas iniciativas ya son consideradas en el informe Horizon (Johnson, 2013) en el plazo máximo de cuatro a cinco años, su mención o reconocimiento como parte de un lenguaje en el campo de la educación mediada por TIC aún es incipiente.

El informe Horizon (Johnson, 2013) en su versión para América Latina es un importante referente para reconocer el proceso de virtualización de la educación en la región. No se

La virtualización de la educación superior en América Latina: entre tendencias y paradigmas. María Elena Chan. 
consideró equiparable al tipo de referentes que fueron contrastados en el ejercicio analítico, por la naturaleza de sus propósitos y objeto de estudio.

La organización NMC News Media Consortium utiliza también el método de consulta a expertos aplicando el Delphi NMC, con el que se ha venido trabajando para identificar consensos en relación al impacto de tecnologías en educación, aprendizaje e investigación creativa. De entre cuarenta tecnologías que presentaron a los expertos, estos eligieron doce, las cuales se distribuyen en diferentes plazos de incorporación en educación. Las tecnologías son entendidas para Horizon en un sentido amplio, no se restringe el término a los aparatos, programas o aplicaciones. El concepto de tecnología abarca métodos, funciones y uso. Así tenemos por ejemplo que las doce tecnologías elegidas para el horizonte 2013-2018 en América Latina fueron:

- Para un año o menos: aprendizaje en línea, contenido abierto, entornos colaborativos y redes sociales.

- Para dos a tres años: analíticas de aprendizaje, aprendizaje móvil, aprendizaje personalizado y realidad aumentada.

- Para cuatro a cinco años: aprendizaje automático, impresión 3D, internet de las cosas y laboratorios virtuales y remotos.

En algunos de los términos utilizados para nombrar las tecnologías se alcanza a identificar algún tipo de postura teórica inherente al calificativo o adjetivo que se utiliza. Es el caso por ejemplo de: aprendizaje móvil, contenido abierto, entornos colaborativos, aprendizaje personalizado. Estos conceptos aunque puedan tener múltiples acepciones, se encuentran ya situados en constructos teóricos o movimientos organizados. Pero no sucede lo mismo con todos los términos utilizados, pues la denominación se centra en el tipo de dispositivo o aplicación sin que el concepto lleve a pensar en modelos educativos: realidad aumentada, impresión 3D, internet de las cosas o redes sociales, pueden ser tecnologías asociadas a cualquier corriente pedagógica, y también a cualquier práctica social no necesariamente educativa.

Ocho de las doce tecnologías elegidas para el informe Horizon tienen en común su condición espacial, o su sentido de puente entre lo real y lo virtual: entornos colaborativos, redes sociales, aprendizaje móvil, personalizado, realidad aumentada, impresión 3D, el internet de las cosas y los laboratorios virtuales y remotos.

En las tecnologías que reporta el informe Horizon es evidente que se confiere importancia a aquellas que permiten interacción, continuidad entre espacios reales y virtuales, así como experiencias enriquecidas por sus posibilidades de representación y creación. Habría que identificar el tipo de interpretación que formadores y gestores dan al informe y el modo como se retroalimentan las creencias y opiniones que contribuyen a la construcción del campo de la educación mediada por TIC y el proceso mismo de virtualización de la educación a partir de la diseminación de las tendencias.

\section{Virtualización educativa y construcción de futuro}

Otro referente indispensable al hablar de virtualización de la educación en América Latina es la obra que ha venido realizando Claudio Rama Vitale desde diferentes instancias de observación: como encargado en su momento del IESALC Instituto Internacional para la Educación Superior en América Latina y el Caribe (UNESCO), el Observatorio de la Educación Virtual de la organización Virtual Educa (iniciativa de la

La virtualización de la educación superior en América Latina: entre tendencias y paradigmas. María Elena Chan. 
OEA) y como co-autor de diversos estudios colaborativos sobre tendencias institucionales en educación superior y en educación a distancia, algunas presentadas como referentes del análisis comparativo realizado para este artículo (Morocho \& Rama, 2012) (Lupion Torres \& Rama, 2010).

La investigación de Claudio Rama referida a la Reforma de la virtualización de la Universidad (2012), propone un modelo epistémico, teórico y metodológico para abordar el análisis de tendencias que interesa resaltar en este artículo.

Claudio Rama (2012) problematiza el contexto regional desde una visión económicosocial de la tecnología, y explica la virtualización de los aprendizajes desde la lógica de cambio del contexto laboral y de los modos de producción de conocimiento. Esto es significativo, porque al igual que el informe del (SITEAL, 2014), la virtualización se observa como componente central del contexto socio-económico y cultural y actúa como megatendencia. Esta visión permite que la reflexión sobre la mediación de las TIC en la educación rebase el sentido instrumental y didáctico, centrado en los intereses de las instituciones educativas y coloque la virtualización como un proceso histórico más amplio.

Con base en un estudio anterior realizado en el 2009, Claudio Rama identificó seis Macrotendencias que estaban impactando a la educación superior en América Latina: Masificación, Regulaciones, Mercantilización, Diferenciación, Internacionalización y Virtualización. El modelo analítico incluye la consideración de tensiones que se generan por cada Macrotendencia y sus respectivos impulsores externos. En la publicación del 2012, retoma el modelo para analizar las Macrotendencias pero tomando como referente a la Educación a Distancia.

El modelo de análisis tiene enorme potencial heurístico. Le permitió reconocer por ejemplo, dinámicas como la tensión entre diferenciación y des-homogeneización de la educación superior respecto a la educación a distancia. Lo que observó fue la creciente variación entre modelos, mayor diversidad de la oferta educativa. El panorama de alta flexibilidad y diversidad torna compleja la regulación, la evaluación e incluso la comparación entre lo que ofrecen las instituciones. A su vez reconoció seis tendencias en el ámbito de la virtualización de la educación: hibridización, multimodalidad pedagógica, fraccionamiento, diferenciación, complejización y consorciación.

La aplicación del modelo analítico de Rama (2012) posibilita reconocer puntos de articulación entre las tendencias y los paradigmas en el campo de la educación virtual:

La virtualización de la educación superior en América Latina: entre tendencias y paradigmas. María Elena Chan. 


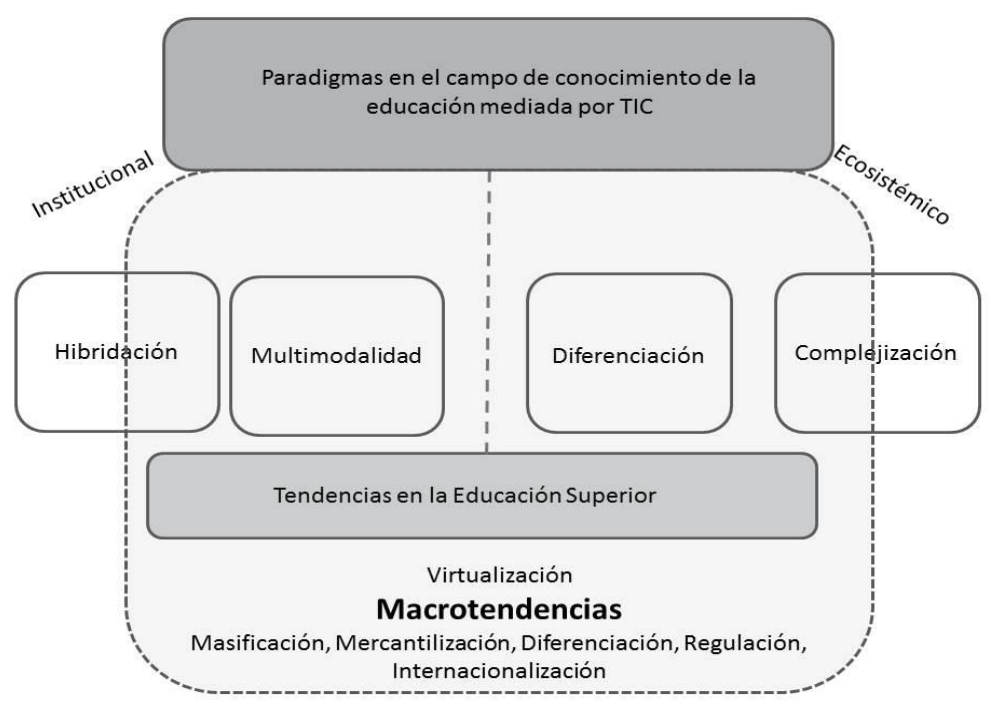

Figura 2. Puntos de articulación entre tendencias y paradigmas en el campo de la educación mediada por TIC

Rama (2012) afirma que la virtualización es una de las macrotendencias dominantes en la Educación Superior en los últimos años. En la figura (2) se muestran cuatro de las tendencias que identificó como predominantes en la educación a distancia en su devenir como digital o virtual. Se han colocado en la figura como mediación entre las tendencias de gestión y políticas, tales como la consorciación, la estandarización, y acreditación. Este tipo de tendencias aunque se nutren de los paradigmas que fundamentan los modelos, no tienen el mismo grado de articulación con el campo de conocimiento de la educación mediada por TIC, que las cuatro mostradas al centro.

A su vez se considera que la hibridación de modelos, recursos y medios, así como la diversificación de las modalidades en tránsito a la multimodalidad, requieren de desarrollo a través de la formulación teórica y metodológica. Se han colocado del lado del paradigma institucional, porque forman parte de la transformación de las organizaciones escolares, sus regulaciones, y gestión de la calidad.

En cambio las tendencias de diferenciación y complejización, tienen una naturaleza que requiere del planteamiento de nuevos problemas, métodos y marcos teóricos para la investigación y desarrollo. Tanto la hibridación como la complejización, suponen una trama de entornos, medios, procesos de adaptación, interacciones, encuentro con lo diverso, y son por ello tendencias que pueden alinearse con un paradigma ecosistémico.

Las tendencias de hibridación, multimodalidad, diferenciación y complejización, significan un puente entre la gestión y la producción de conocimiento, porque requieren de modelos y fundamentos epistémicos emergentes. La diferenciación y la complejización son tendencias opuestas, de alguna manera, a la regulación y la estandarización, por ello se consideran menos institucionales y más abiertas a los nuevos modos de educar en la virtualidad.

"De igual forma que nadie, cuando aparecieron la Mecánica Cuántica o la Teoría de la Relatividad, la contrapuso con la mecánica clásica de Newton y de Leibnitz, sino que quedó integrada como una aplicación local de unas teorías más generales, es de esperar que algo parecido suceda con las investigaciones y los desarrollos teóricos existentes y

La virtualización de la educación superior en América Latina: entre tendencias y paradigmas. María Elena Chan. 
los nuevos, que supondrán una continuidad en la línea de adaptación de las estrategias docentes, de la ayuda pedagógica, a las nuevas condiciones. De manera que se utilicen para poner los bienes del conocimiento, los OER y los contenidos de los MOOCs, cerca de las distintas formas de aprender y de integrar los conocimientos en los distintos esquemas y en las representaciones individuales, posibilitar que los alumnos elaboren a partir de ellas, y que sean transferidas en contextos diferentes, atribuyéndoles sentido. En definitiva posibilitar que se aprenda en los nuevos contextos." (Zapata-Ros, 2013, pág. 35)

Para entender el fenómeno de la virtualización de la educación superior, probablemente haya que reconocer como sistema emergente uno nuevo surgido del choque entre el paradigma instrumental (pedagógico-mediático) centrado en la institución escolar, con el paradigma ecosistémico, abierto y orientado al desarrollo tecnocultural, organizado fuera del contexto escolar, con una dinámica promovida por los mismos medios de comunicación digitales.

El tipo de análisis prospectivos suelen centrarse en las prácticas institucionales. Es comprensible que la mayoría de los expertos en EAD y elearning se encuentran en organizaciones escolares y ven en estas modalidades ventajas sobre la educación presencial convencional. Los investigadores que dedican su tiempo al estudio del fenómeno están interesados en lo que está sucediendo con sus propias instituciones. En cambio, los que están operando innovaciones fuera de la escuela están ocupados en el hacer, en el documentar y difundir, y generan una gran cantidad de información que no necesariamente está circulando en las redes académicas, porque no siguen sus reglas, utilizan blogs, redes sociales y otras estrategias novedosas que muy pocos académicos están operando para fortalecer su red.

La captación de estos fenómenos es indispensable para completar el mapa de tendencias y reconfiguraciones paradigmáticas. Posiblemente se generen algunas tensiones entre los modelos educativos institucionalizados con la etiqueta de educación "a distancia" y los que se están transformando paulatinamente, a partir de la emergencia de prácticas culturales que tienden hacia el aprendizaje y la enseñanza abierta, ubicua y centrada en el aprender haciendo.

Se diría que hay una innovación inducida y otra emergente, una innovación de los procesos instituidos, y otra que está transformando los ecosistemas de conocimiento más allá de las escuelas.

El logro de la mayor calidad en el diseño educativo, la organización escolar, la cooperación internacional y el dominio de la formación virtual como ruta conocida y relativamente segura para lograr mayor equidad educativa, tiene amplio consenso entre los especialistas en educación mediada por TIC, y se mantiene en una dinámica de fortalecimiento continuo, gracias a los esfuerzos de entidades como las que se han presentado en este ejercicio, que observan y actúan a favor de la cooperación internacional. Esos esfuerzos deben sostenerse, particularmente aquellos que abren un espacio a la interacción permanente de actores clave que actúan como sensores de lo que está sucediendo en el campo de la educación mediada por tecnologías, como los foros de la Cátedra UNESCO de Educación a Distancia, el Observatorio de Virtual Educa, del CAEU/OEI y organizaciones como el SITEAL. El modelo utilizado por este sistema parece particularmente fecundo para la comprensión del fenómeno de la virtualización educativa desde una perspectiva integral.

La virtualización de la educación superior en América Latina: entre tendencias y paradigmas. María Elena Chan. 
La otra vía, la de la innovación educativa no instituida, la que se está basando en los ecosistemas de conocimiento y comunicación que trascienden las organizaciones escolares, tal vez presenten menos problemas para crecer porque no les afectan las regulaciones, ni las inercias, no cargan con el peso de lo escolar instituido. Se difunden y colocan como tendencia con mayor velocidad porque manejan canales de comunicación basados en redes sociales y el impacto parece multiplicarse.

Sin embargo, por ahora la información sobre las experiencias extraescolares es casuística, no está sistematizada a gran escala, y sus protagonistas apenas asoman al ámbito escolar convidados para llevar a cabo algunas experiencias temporales. Retomando la metáfora de Dolores Reig (SITEAL, 2014), la cinta de Moebius puede ilustrar lo que está por venir en cuanto a un movimiento creciente de virtualización educativa. Probablemente la mayor fuerza de cambio se encuentre en la transformación cultural de los educandos, y haya que generar nuevos indicadores para medir el aprendizaje en términos de competencias ciberculturales, entendidas éstas como la capacidad de gestionar información, gestionar conocimiento, comunicar y participar en la inteligencia colectiva.

Presentación del artículo: 18 de diciembre de 2015

Fecha de aprobación: 26 de diciembre de 2015

Fecha de publicación: 30 de enero de 2016

Chan, M.E. (2016). La virtualización de la educación superior en América Latina: entre tendencias y paradigmas. RED. Revista de Educación a Distancia. 48(1). Consultado el (dd/mm/aaaa) en http://www.um.es/ead/red/48

\section{Bibliografía}

Adell, J., \& Castañeda, L. (2013). Los entornos de aprendizaje PLEs: una nueva manera de entender el aprendizaje. En L. Castañeda Quintero, \& J. Adell Segura, Entornos personales de aprendizaje: claves para el ecosistema educativo en red. (pág. 192). Alcoy: Marfil.

Arnold Cathalifaud. (2004). La construcción del conocimiento: Fundamentos epistemológicos del constructivismo autopoiético. Investigaciones sociales, 271-287.

Avila, P. (2012). Educación a distancia y educomunicación. En M. M. Castañeda, Veinte visiones sobre la educación a distancia (págs. 233-246). Guadalajara: UDGVIRTUAL.

Bates, T. (12 de Octubre de 2014). Online learning and distance education resources.

Recuperado el 27 de Noviembre de 2015, de What is MOOC?: http://www.tonybates.ca/2014/10/12/what-is-a-mooc/

Blois, M. (2012). A trajetória de uma vida dedicada à educação a distancia.Minha visão da Educação à Distância ao longo de quatro. En M. C. (coord), Veinte visiones sobre la educación a distancia (págs. 111-130). Guadalajara: UDGVIRTUAL.

Bourdieu, P. (1994). Razones prácticas sobre la teoría de la acción. París: Seuil.

La virtualización de la educación superior en América Latina: entre tendencias y paradigmas. María Elena Chan. 
Cabero, J. (2012). La educación a distancia hacia el e-learning 2.0:. En M. M. Castañeda, Veinte visiones sobre la educación a distancia (págs. 247-263). Guadalajra: UDGVIRTUAL.

Cabero, J., \& Vázquez, I. (2013). Los entornos personales de aprendizaje: uniendo lo formal, lo informal y no formal para la construcción de conocimiento. En F. S. Cecilia, \& M. Fonseca Sardí, Entornos personales de aprendizaje: visiones y retos para la formación (pág. 121). Caracas: Universidad Metropolitana.

Cembrano, D. (2010). Una educación personalizante, proyecto pedagógico para el siglo XXI. Cuadernos Educación 23. Santiago, Chile: Universidad Alberto Hurtado.

Chan Núñez, M. (2006). Investigación de la educación virtual. Guadalajara: INNOVA, Universidad de Guadalajara.

Chan, M. E. (2015). De las plataformas a los ambientes educativos personalizados. En R. C. Judith, La educación a distancia en México, una nueva realidad universitaria. México: ECOESAD / VIRTUAL EDUCA.

de Zubiría Samper, J. (2008). De la escuela nueva al constructivismo. Un análisis crítico. Bogotá: Aula abierta. Magisterio.

Downes, S. (2012). Connectivism and connective knowledge,. National Research Canadian Cuncil.

Echeverría, J. (2000). Un mundo virtual. Barcelona: Plaza y Janés.

Editacuja. (2015). Editacuja. Recuperado el 28 de Noviembre de 2015, de AppiarioLaboratorios de aprendizaje transmedia para le mundo real: http://www.editacuja.com.br/

Flipped Learning Network. (2014). The Four Pillars of F-L-I-P. Recuperado el 28 de Noviembre de 2015, de www.flippedlearning.org/definition: www.flippedlearning.org/definition

García Aretio, L. (2012). Criterios teóricos para alimentar la práctica en educación a distancia. En M. Manuel, Veinte visiones sobre la educación a distancia (págs. 53-68). Guadalajara: UDGVIRTUAL.

García Aretio, L., Ruiz corbella, M., Quintana Díaz, J., García Blanco, M., \& García Pérez, M. (2009). Concepción y tendencias de la educación a distancia en América Latina. Madrid: CAEU OEI, AECID.

Giraldo, M. E. (2012). Enfoque pragmático de la comunicación como opción para pensar los ambientes virtuales de aprendizaje. En M. Manuel, Veinte visiones sobre la educación a distancia (págs. 263-282). Guadalajara: UDGVIRTUAL.

Habermas, J. (1989). Teoría de la Acción Comunicativa,. Madrid: Taurus.

La virtualización de la educación superior en América Latina: entre tendencias y paradigmas. María Elena Chan. 
Hannafin , M., Land, S., \& Oliver, K. (2000). Entornos de aprendizaje abiertos: fundamentos, métodos y modelos. En R. Carles, Teorías y modelos de la instrucción. Madrid: Santillana.

Johnson, L. A. (2013). NMC Perspectivas tecnológicas. Educación Superior en América Latina 2013-2018. Un análisis regional del Informe Horizon NMC. Austin, Texas: The News Media Consortium.

Jonassen, D. (2000). El diseño de entornos constructivistas de aprendizaje, . En Reigeluth, Diseño de la instrucción, Teorías y modelos, Parte I. Aula XXI, Santillana.

Kuhn, T. (1962). Kuhn, Thomas S. (1971) La estructura de las revoluciones científicas. . México, D.F.: Fondo de Cultura Económica.

Kuhn, T. (1971). La estructura de las revoluciones cientificas. México: Fondo de Cultura Económica.

Lupion Torres, P., \& Rama, C. (2010). La educación superior a distancia en América Latina y el Caribe. Palhoca: UNISUL.

Martín Barbero Jesús, (. (2000). Jóvenes, comunicación e identidad, en:. OEI - Cumbres Cultura - Conferencia 2000. Panamá: Organización de Estados Americanos para la educación, la ciencia y la cultura.

Mateos, R., García, M. F., \& González, M. I. (2015). Megatendencias: aproximaciones al campo de la educación. En M. E. Chan, \& coord., Educaión y cultura en ambientes virtuales (págs. 5-26). Guadalajara: UDGVIRTUAL.

Maturana, H. (1997). Instituto de Terapia Cognitiva. Recuperado el 07 de septiembre de 2015, de Artículos y conferencias: Metadesign: http://www.inteco.cl/articulos/006/texto_ing.htm

Miklos, T., \& Arroyo, M. (2008). Una visión prospectiva de la educación a distancia en América Latina. Universidades, 49-67.

Morales Gómez, G. (26 de Abril de 2013). Pedagogía. Recuperado el 29 de Noviembre de 2015, de PARADIGMAS-ENFOQUES-MODELOS-COParadigmas, efoques, modelos, corrientes y tendencias pedagógicas: http://pfc2normal.blogspot.mx/2013/04/buenas-nochesapreciados-estudiantes.html

Moreno Castañeda, M. (2012). Veinte visiones sobre la educación a distancia. Guadalajara: UDGVIRTUAL.

Morocho, M., \& Rama, C. (2012). Las nuevas fronteras de la educación a distancia. Loja: Virtual Educa / UTPL.

Pata, K. (2009). Revising the framework of knowledge ecologies: how activity patterns define. En L. \&. Romero, Educational Social Software for Context-Aware Learning: Collaborative (págs. 241-267). New York: n. Information Science Reference. Hershey. .

La virtualización de la educación superior en América Latina: entre tendencias y paradigmas. María Elena Chan. 
Peters, O. (1993). La educación a distancia y la producción industrial. una interpretación comparativa en contorno. En D. K. D. Sewart, Educación a Distancia: Perspectivas Internacionales (págs. 95-113). Londres y Nueva York: Routledge Croom Yelmo.

Piaget, J. (1978). Adaptación vital y psicología de la inteligencia. Madrid: Siglo XXI.

Pogrow, S. (2000). El uso sistemático de entornos de alta estimulación para acelerar el aprendizaje de los alumnos menos aventajados de los cursos de $4 \mathrm{o}$ de primaria a $2 \mathrm{do}$ de secundaria. En R. Carles, Teorías y modelos de la instrucción. Madrid: Santillana.

Rama, C. (2012). La reforma de la virtualización de la Universidad. Guadalajara: UDGVIRTUAL.

Reigeluth, C. (2000). Diseño de la instrucción: teorías y modelos. Un nuevo paradigma de la teoría de la instrucción. Madrid: Santillana.

Reigeluth, C. (2012). Teoría instruccional y tecnología para el nuevo paradigma de la. Revista de educación a distancia No. 32.

Sarramona, J. (2012). La educación a distancia vista en perspectiva. En M. M. Castañeda, Veinte visiones sobre la educación a distancia (págs. 283-296). Guadalajara: UDGVIRTUAL.

SITEAL. (2014). Informe sobre tendencias sociales y educativas en América Latina. Buenos Aires: UNESCO, OEI, SITEAL, .

Soares, O. d. (1999). "Comunicacao/Educacao: a emergencia de um novo campo o perfil de seus profissionais". Contrato No.2, 5-75.

Stanford University. (2015). Transformative Learning Technologies Lab. Recuperado el 25 de Noviembre de 2015, de https://tltl.stanford.edu/projects/fablabschool

Victoria, M. (2012). Visión de la educación en red más allá de la distancia. En M. M. Castañeda, Veinte visiones sobre la educación a distancia (págs. 131-148). Guadalajara: UDGVIRTUAL.

Vigotsky, L. (1978). Pensamiento y lenguaje. Madrid: Paidós.

Visser, J. (2012). La innovación: necesidad científica, opción artística. En M. C. Manuel, Veinte visiones de la educación a distancia (págs. 31-52). Guadalajara: UDGVIRTUAL.

Yee Seuret, M. (2012). La educación a distancia como un bien público: una visión. En M. C. Manuel, Veinte visiones sobre la educación a distancia (págs. 95-110). Guadalajara: UDGVIRTUAL.

Zafra, E. (s.f.). Innovadores menores de 35 años. MIT Technology Review.

Zapata-Ros, M. (2013). MOOCs, una visión crítica y una alternativa complementaria: La individualización del aprendizaje. Campus Virtuales, 20-38.

La virtualización de la educación superior en América Latina: entre tendencias y paradigmas. María Elena Chan. 
Zapata-Ros, M. (2015). Teorías y modelos sobre el aprendizaje en entornos conectados y ubicuos. Bases para un nuevo modelo teórico a partir de una visión crítica del conectivismo. Education in the Knowledge Society, 69-102.

La virtualización de la educación superior en América Latina: entre tendencias y paradigmas. María Elena Chan. 